TRANSACTIONS OF THE

AMERICAN MATHEMATICAL SOCIETY

Volume 355, Number 3, Pages 935-965

S 0002-9947(02)03076-3

Article electronically published on October 29, 2002

\title{
THE DOUBLE OF A HYPERBOLIC MANIFOLD AND NON-POSITIVELY CURVED EXOTIC $P L$ STRUCTURES
}

\author{
PEDRO ONTANEDA
}

\begin{abstract}
We give examples of non-compact finite volume real hyperbolic manifolds of dimension greater than five, such that their doubles admit at least three non-equivalent smoothable $P L$ structures, two of which admit a Riemannian metric of non-positive curvature while the third does not. We also prove that the doubles of non-compact finite volume real hyperbolic manifolds of dimension greater than four are differentiably rigid.
\end{abstract}

\section{INTRODUCTION}

Some of the most interesting aspects of the relationship between geometry and topology are the rigidity theorems. Among the most well known are, for instance, Bieberbach's Theorem: two homotopically equivalent flat manifolds are affinely isomorphic (hence diffeomorphic), or Mostow's Theorem: two homotopically equivalent closed hyperbolic $n$-manifolds are isometric (hence diffeomorphic), for $n>2$, or Farrell-Jones' Topological Rigidity Theorem: two homotopically equivalent closed $n$-manifolds, one of which is non-positively curved, are homeomorphic, for $n>4$ ("curvature" means "sectional curvature"). On the other hand, Farrell and Jones also gave counterexamples to "non-positive curvature implies differentiable rigidity". In fact, they gave counterexamples to "strictly negative curvature implies differentiable rigidity" (known as the Lawson-Yau conjecture), in dimensions larger than six (see [7]). In dimension six, counterexamples appear in [15, and in dimensions less than six, the question remains open.

Thus we have that non-positive curvature implies topological $(n>4)$ but not differentiable $(n>5)$ rigidity. Also, the results in 15 and [10 show that nonpositive curvature (in fact, even negative curvature) does not imply $P L$ rigidity either. That is, there are examples of negatively curved closed Riemannian $n$ manifolds, $n>5$, that are homeomorphic but not $P L$ homeomorphic.

On the other hand, there is a simple example of an $n$-manifold, $n>4$, with many differentiable structures (some of them inducing different $P L$ structures) such that only one differentiable structure admits a non-positively curved Riemannian metric. This manifold is the $n$-torus, and the result just mentioned follows from the torus theorem of Lawson and Yau [13] and Gromoll and Wolf [11].

Now, if $M$ is one of the examples mentioned above (i.e., $M$ has different negatively curved $P L$ structures), we have that $M \times T^{m}$ has many different $P L$ structures, some non-positively curved and some not: we can change the $P L$ structure

Received by the editors April 12, 2001 and, in revised form, April 12, 2002.

2000 Mathematics Subject Classification. Primary 53C20, 57Q25, 57R55.

This research was supported in part by CNPq, Brazil. 
on $M$ so that we get different non-positively curved $P L$ structures on $M \times T^{m}$; if we change the $P L$ structure on $T^{m}$, it is not difficult to see that we get $P L$ structures that do not admit non-positively curved $P L$ structures. Still, these examples are too artificial, because there is too much zero curvature. In this paper, we get better examples with much less zero curvature. For this we study the double of a non-compact finite volume real hyperbolic manifold.

A non-compact finite volume real hyperbolic $n$-manifold is homeomorphic to the interior of a compact manifold with boundary. Each boundary component is a flat manifold of dimension $n-1$. The compact manifold with boundary can be doubled along its boundary to form $D M$, the double of $M$. It is well known that the hyperbolic metric can be modified on each cusp (see section 1) to produce a metric of non-positive curvature. Here we construct examples of non-compact finite volume real hyperbolic manifolds of dimension greater than five, such that their doubles admit at least three non-equivalent smoothable $P L$ structures, two of which admit a Riemannian metric of non-positive curvature while the other does not.

Explicitly, here is the statement of our main theorem:

Theorem 1. There are examples of non-compact finite volume real hyperbolic $n$ manifolds $M, n>5$, such that $\Sigma_{3}$

(i) DM has, at least, three non-equivalent (smoothable) PL structures $\Sigma_{1}, \Sigma_{2}$,

(ii) $\Sigma_{1}$ and $\Sigma_{2}$ admit a Riemannian metric with non-positive curvature, and negative curvature outside a hypersurface.

(iii) $\Sigma_{3}$ does not admit a Riemannian metric with non-positive curvature.

In fact, $\Sigma_{3}$ does not even admit a piecewise flat metric of non-positive curvature.

At this point we think it is interesting to mention a result, which is of independent interest, that we needed during the proof of the main theorem (to show that non-concordant $P L$ structures are, in fact, not $P L$ equivalent, see corollary 9.6). This result tells us that the doubles of non-compact finite volume real hyperbolic manifolds are differentiably rigid.

Theorem 2. Let $f: D M \rightarrow D M$ be a homeomorphism, where $D M$ is the double of a non-compact finite volume real hyperbolic $n$-manifold $M, n \geq 3$. Then $f$ is homotopic to a diffeomorphism $g: D M \rightarrow D M$.

The proof of this theorem actually proves also:

Theorem 2A. Let $f: D M_{1} \rightarrow D M_{2}$ be a homotopy equivalence, where $D M_{1}$ and $\mathrm{DM}_{2}$ are the doubles of non-compact finite volume real hyperbolic $n$-manifolds, $n \geq 5$. Then $f$ is homotopic to a diffeomorphism $g: D M_{1} \rightarrow D M_{2}$.

The proof of Theorem 2A uses Mostow's Rigidity Theorem and results of Farrell and Jones. We now return to the discussion of our main theorem. Theorem 1 follows from Theorems 3, 4, 5 and 6 below. Theorem 3 gives conditions for a construction of doubles with non-equivalent smoothable $P L$ structures that admit Riemannian metrics of non-positive curvature. Also, Theorem 4 gives conditions for a construction of doubles with $P L$ structures that do not admit a Riemannian metric of non-positive curvature.

Theorem 3. Let $M$ be a non-compact finite volume hyperbolic $n$-manifold, $n>5$, with a large cusp $C_{M}$ diffeomorphic to $T^{n-1} \times(0, \infty)$. Assume either 
(1) $n$ is odd, or

(2) $n$ is even and there is a totally geodesic hypersurface $N \subset M$, with exactly one cusp contained in the cusp $C_{M}$ of $M$.

Then the double DM of $M$ admits (at least) two different (smoothable) PL structures admitting Riemannian metrics with non-positive curvature.

For the definition of large cusp see 2.12, and to understand what we mean by "a cusp contained in a cusp" see 4.3 .

Theorem 4. Let $M, N$ be two non-compact finite volume real hyperbolic manifolds such that

(1) $N$ is a totally geodesic submanifold of $M$,

(2) $\operatorname{dim} M=n \geq 5, \operatorname{dim} N=n-3$,

(3) the normal bundle of $N$ is trivial,

(4) $M$ has a cusp $C_{M}$ diffeomorphic to $T^{n-1} \times(0, \infty)$,

(5) $N$ has exactly one cusp contained in $C_{M}$.

Then the double $D M$ of $M$ has a differentiable structure $\mathcal{E}$, such that

(i) $(D M, \mathcal{E})$ is not $P L$ equivalent to $D M$, and

(ii) $(D M, \mathcal{E})$ does not admit a Riemannian metric with non-positive curvature.

In fact, DM does not even admit a piecewise flat metric with non-positive curvature.

Note that in both theorems we demand the non-compact finite volume real hyperbolic $n$-manifold $M$ to have a cusp diffeomorphic to $T^{n-1} \times(0, \infty)$ (the existence of such manifolds is granted by theorem 5 below).

The idea of the proof of theorem 3 is to reglue the cusp (the one diffeomorphic to $\left.T^{n-1} \times(0, \infty)\right)$ using an exotic diffeomorphism of $T^{n-1}$. If the cusps are "large", the smooth structure obtained by this regluing admits a Riemannian metric of non-positive curvature. This metric agrees with the original hyperbolic metric on a complement of a collared neighborhood of $T^{n-1}$. To prove that the $P L$ structure induced by the smooth structure obtained by the regluing is not $P L$ equivalent to the original one, we just have to prove, by a corollary of theorem 2 (see corollary 9.6) that this $P L$ structure is not concordant to the original one. But recall that, by results of Kirby and Siebenmann, the set of concordant classes of $P L$ structures on a $P L$ manifold of dimension greater than four is in bijective correspondence with its third cohomology group with coefficients in $\mathbb{Z}_{2}$. In view of this, it is not difficult to prove (see section 3) that there is a bijection between the cohomology classes corresponding to the $P L$ structures obtained by this type of regluing and the image of the natural map $\iota_{3}: H^{3}\left(D M, D M \backslash T^{n-1}\right) \rightarrow H^{3}(D M)$. Hence we need $\iota_{3} \neq 0$. It turns out (see lemma 4.4 and remark 4.5) that $\iota_{3}$ is never zero for $n$ odd. If $n$ is even $\iota_{3} \neq 0$ if there is a codimension one, totally geodesic submanifold $N$ such that the given cusp of $M$ "contains exactly one cusp of $N$ ". Note that these two conditions are exactly (1) and (2) of the statement of theorem 3.

The idea of the proof of theorem 4 is the following. Let $N$ be a totally geodesic submanifold of codimension 3 in $M$ with trivial normal bundle. Suppose that $N$ intersects the cross section of the given cusp in a homologically non-trivial fashion. Let $x \in H^{3}(D M)$ be the cohomology class dual to $[D N] \in H_{n-3}(D M)$. Then we will prove that it is a consequence of the Flat Torus Theorem that the $P L$ structure corresponding to $x$ does not admit a Riemannian metric of non-positive curvature. 
It remains to prove that there are non-compact finite volume real hyperbolic manifolds with the desired properties. For this we use a standard method, following ideas of J. J. Millson. Indeed, let $\mathcal{Q}$ be a rational quadratic form of signature $(n, 1)$, $n \geq 5$. Then, for certain integers $a$, the pair $(\mathcal{Q}, a)$ determines a non-compact finite volume real hyperbolic $n$-manifold, $n \geq 5$, that we denote by $M(\mathcal{Q}, a)$ (these manifolds are arithmetically defined real hyperbolic manifolds; see section 7$)$.

For every integer $b, M(\mathcal{Q}, b a)$ is a finite cover of $M(\mathcal{Q}, a)$.

The next theorem says that these arithmetically defined non-compact finite volume real hyperbolic manifolds have finite covers with cusps having tori as cross sections:

Theorem 5. If $M(\mathcal{Q}, a)$ is as above, then it admits finite covers $M(\mathcal{Q}$, ba) having one cusp diffeomorphic to $T^{n-1} \times(0, \infty)$.

In fact, $M(\mathcal{Q}, a)$ admits finite covers $M(\mathcal{Q}, c a)$ with all cusps diffeomorphic to $T^{n-1} \times(0, \infty)$ (corollary 7.3). Also, there is a corollary of the proof of theorem 5 which says that, by taking further congruence subgroups (i.e., $M(\mathcal{Q}, q b a)$, for some $q$ ), we can assume the cusp to be large.

Finally, we prove that we can find manifolds satisfying conditions (1) or (2) of theorem 3, and (1)-(5) of theorem 4 . We will require that the quadratic form $\mathcal{Q}$ satisfies:

0.1. $\mathcal{Q}$ has the form $\mathcal{Q}\left(x_{1}, \ldots, x_{n+1}\right)=\lambda_{1} x_{1}^{2}+\ldots+\lambda_{n} x_{n}^{2}-\lambda_{n+1} x_{n+1}^{2}$, with $\lambda_{i}>0$, $\lambda_{i} \in \mathbb{Q}$, and $\lambda_{1}=\lambda_{2}=\lambda_{3}$. Also, if $n \leq 6, \lambda_{4} x_{4}^{2}+\ldots+\lambda_{n} x_{n}^{2}-\lambda_{n+1} x_{n+1}^{2}$ has a rational isotropic vector.

Theorem 6. Let $M(\mathcal{Q}, a)$ be as before with $\mathcal{Q}$ satisfying $(0.1)$. Then $M(\mathcal{Q}, a)$ admits finite covers $M_{0}$, such that there are non-compact finite volume real hyperbolic manifolds $M_{1}, M_{2}$ satisfying the following conditions:

(1) $\operatorname{dim} M_{0}=n \geq 5, \operatorname{dim} M_{1}=n-1, \operatorname{dim} M_{2}=n-3$;

(2) $M_{2} \subset M_{1} \subset M_{0}$ and the inclusions are totally geodesic;

(3) the normal bundle of $M_{2}$ in $M_{0}$ is trivial;

(4) $M_{0}$ has a large cusp $C_{M_{0}}$ diffeomorphic to $T^{n-1} \times(0, \infty)$;

(5) $M_{i}$ has exactly one cusp contained in $C_{M_{0}}, i=1,2$.

The finite covers obtained are not necessarily of the form $M(\mathcal{Q}, a)$.

We remark that in 1] examples are given of doubles of non-compact finite volume hyperbolic manifolds with exotic differentiable structures not admitting a nonpositively curved metric. In these examples the $P L$ structure does not change.

For other related results see [9], where examples are given of non-compact finite volume hyperbolic manifolds with exotic differentiable structures not admitting a pinched negatively curved metric.

The paper is structured as follows. In section 1 we define the double of a noncompact finite volume hyperbolic manifold. In section 2 we give some geometric lemmas that will allow us to provide exotic triangulations with a non-positively curved metric. In this section we also discuss large cusps. In section 3 we state some triangulation lemmas that we will use to change triangulations. Section 4 has more lemmas and remarks. In section 5 we prove theorem 3 . In section 6 we prove theorem 4 . Theorem 5 is proven in section 7 and theorem 6 in section 8 . We prove theorem 2 in section 9. Finally, we have an appendix in which we prove a technical lemma. 
We are very grateful to the referee for pointing out several corrections and improvements to the text. Some portions of the introduction above were inspired by his report.

\section{The Double}

1.1. Let $M$ be a non-compact finite volume real hyperbolic manifold. Then it has a finite number of cusps, each one isometric to some $F_{i} \times[0, \infty)$ with metric $e^{-2 t} S_{i}+d t^{2}$, where the $F_{i}$ 's are compact connected manifolds with flat metric $S_{i}$ (see [2], sec. D3). In what follows we identify the cusps with $F_{i} \times[0, \infty)$. The double $D M$ of $M$ is obtained by cutting $M$ in the cusps, that is, consider $M \backslash\left\{F_{i} \times\right.$ $(b, \infty)\}$ for some positive real number $b$, and glue two copies along the boundary $F_{i} \times\{b\}$, for all $i$, by the identity map. $D M$ has a unique (up to diffeomorphism) differentiable structure such that its restriction to each copy of $M \backslash\left\{F_{i} \times(b, \infty)\right\}$ is the differentiable structure induced by the hyperbolic structure. This is the canonical differentiable structure on $D M$.

Note that $D M$ can be given a non-positively curved metric in the following way. Given $\epsilon>0$, it is straightforward to find an $f_{\epsilon}: \mathbb{R} \rightarrow \mathbb{R}^{+}$such that $f_{\epsilon}^{\prime \prime} \geq 0$, $f_{\epsilon}(t)=e^{t+b}$ for $t \geq \epsilon$ and $f_{\epsilon}(t)=e^{-t+b}$ for $t \leq-\epsilon$. Then if $N_{i}=F_{i} \times(-c, c), c>\epsilon$, with metric $f_{\epsilon}^{2} S_{i}+d t^{2}$, we have that $N_{i}$ is non-positively curved. To see this, note that $f_{\epsilon}^{2} S_{i}+d t^{2}$ is a warped metric (see [3] p. 23) so that the sectional curvature for the plane at the point $(p, t) \in F_{i} \times(-c, c)$ generated by the orthonormal basis $\left\{\lambda\left(\frac{\partial}{\partial t}\right)_{t}+v_{p}, w_{p}\right\}$, where $v_{p}, w_{p} \in T_{p} F_{i}$, is given by

$$
-\frac{f_{\epsilon}^{\prime \prime}}{f_{\epsilon}} \lambda^{2}-\frac{\left(f_{\epsilon}^{\prime}\right)^{2}}{f_{\epsilon}^{2}}\|v\|^{2}
$$

(see [3, p. 27). So, because each $S_{i}$ is flat and $f_{\epsilon}^{\prime \prime} \geq 0$, we have that $N_{i}$ with metric $f_{\epsilon}^{2} S_{i}+d t^{2}$ is non-positively curved. Then $F_{i} \times(-c,-\epsilon), F_{i} \times(c, \epsilon) \subset N_{i}$ are both isometric to $F_{i} \times(b-(\epsilon+c), b-\epsilon) \subset M \subset D M$; so we can put near $F_{i}=F_{i} \times\{b\} \subset D M$ the metric of $N_{i}\left(F_{i} \times\{b\} \subset D M\right.$ corresponds to $\left.F_{i} \times\{0\} \subset N_{i}\right)$, obtaining in this way a non-positively curved metric on $D M$. Note that we can arrange for the curvature to be zero just in the $F_{i}=F_{i} \times\{b\} \subset D M$, and by making $\epsilon$ small we have curvature -1 outside a small neighborhood of the $F_{i} \times\{b\} \subset D M$.

\section{Geometric Lemmas}

Let $M$ be an $n$-dimensional compact manifold and $I=[a, b], 0 \leq a \leq b$, a closed interval. Then the tangent space of $M \times I$ at $(x, t)$ is isomorphic to $T_{x} M \oplus \mathbb{R}\left(\left.\frac{\partial}{\partial t}\right|_{t}\right)$. Also, denote by $p^{i}, i=1,2$, the projection of $M \times I$ onto $M$ and $I$, respectively.

Here we see that if we have a metric on the model $M \times I$, satisfying certain conditions, then, provided that we stretch and enlarge it enough, we have control over the warped metric. We also need to prove a second lemma, because when we try to fit the model on some manifold, we have to take coverings (to make it large), but these coverings make the model grow at different rates in different directions.

2.1. Now consider Riemannian metrics $A$ on $M \times I$ with the following properties:

(a) For any $v \in T_{x} M, A\left(v, \frac{\partial}{\partial t}\right)=0$.

(b) $A\left(\frac{\partial}{\partial t}, \frac{\partial}{\partial t}\right)=1$.

Equivalently, $A=S_{t}+d t^{2}$, where $S_{t}$ is a metric on $M$ depending on $t$. 
2.2. For any metric $A$ satisfying (2.1), and a function $f: \mathbb{R}^{2} \rightarrow \mathbb{R}^{+}$(write $\alpha$ and $t$ for the variables of $f$ and $f_{\alpha}$ for $f(\alpha, t)$ ) define a new metric $A_{\alpha}$ on $M \times I$, depending on $\alpha$ :

$$
A_{\alpha}(v, w)=f_{\alpha}^{2}(\alpha t) A_{1}(v, w)+\alpha^{2} A_{2}(v, w) \quad v, w \in T_{(x, t)} M \times I
$$

where $A_{i}(v, w)=A\left(p_{*}^{i}(v), p_{*}^{i}(w)\right)=\left(p^{i}\right)^{*} A(v, w)$.

Equivalently, if $A=S_{t}+d t^{2}$, then $A_{\alpha}=f_{\alpha}^{2}(\alpha t) S_{t}+\alpha^{2} d t^{2}$.

2.3. As before, let $f$ be such that for $t \in I$ :

(a) $f_{\alpha}(\alpha t) \rightarrow \infty$, as $\alpha \rightarrow \infty$;

(b) $\left(\frac{f_{\alpha}^{\prime}}{f_{\alpha}}\right)(\alpha t) \rightarrow \ell_{1}<\infty$, as $\alpha \rightarrow \infty$;

(c) $\left(\frac{f_{\alpha}^{\prime \prime}}{f_{\alpha}}\right)(\alpha t) \rightarrow \ell_{2}<\infty$, as $\alpha \rightarrow \infty$.

We will require that these limits are uniform for $t$ on a bounded interval.

Write $k_{0}=\max \left\{\ell_{1}^{2}, \ell_{2}\right\}$, and $k_{1}=\min \left\{\ell_{1}^{2}, \ell_{2}\right\}$.

2.4. Lemma. Let $A$ be a Riemannian metric on $M \times I$ satisfying (2.1) and $f$ as in (2.3). Then given $\epsilon>0$, there is an $L$ such that, for $\alpha>L$,

$$
-k_{0}-\epsilon<K\left(A_{\alpha}\right)<-k_{1}+\epsilon,
$$

i.e., all sectional curvatures of $A_{\alpha}$ lie in the interval $\left(-k_{0}-\epsilon,-k_{1}+\epsilon\right)$.

The proof is the same as lemma 3.5 of [7], but there is a proof in the appendix, because we will need some details afterwards.

2.5. Note that the constant $L$ of the lemma depends only on $C$ (see (a.2) of the appendix); so if $A^{\prime}$ is any other metric on $M \times I$ satisfying (2.1) and such that (a.2) is true for some $C^{\prime} \leq C$, then the lemma holds for $A^{\prime}$ with the same $L$.

2.6. When $M$ is the $n$-torus $T^{n}$, we can say a little more than lemma 2.4 , but first some definitions.

If $M$ is parallelizable with $X=\left(X_{1}, \ldots, X_{n}\right), n$ linearly independent globally defined vector fields, then any Riemannian metric $A$ expressed in this basis induces a smooth function $A_{X}: M \rightarrow \mathcal{P}$, where $\mathcal{P}$ is the space of symmetric positive definite $n$ by $n$ matrices. Now given any $\varphi \in G L(n, \mathbb{R})$ (this is the space of invertible $n$ by $n$ real matrices), we can change $A$ to a metric $A^{\varphi}$, by defining (note that the definition depends on $X$ )

2.7. $A_{X}^{\varphi}=\varphi^{\mathrm{T}} \cdot A_{X} \cdot \varphi$ (the $\mathrm{T}$ stands for transpose).

2.8. Now when $M=T^{n-1}=\mathbb{S}^{1} \times \ldots \times \mathbb{S}^{1}$, consider $X^{\prime}=\left\{\frac{\partial}{\partial x_{1}}, \ldots, \frac{\partial}{\partial x_{n-1}}\right\}$ and $X=$ $\left\{\frac{\partial}{\partial x_{1}}, \ldots, \frac{\partial}{\partial x_{n-1}}, \frac{\partial}{\partial t}\right\}$, the canonical globally defined vector fields on $T^{n-1}$ and $T^{n-1} \times$ $I$, respectively. Note that these vector fields come from locally defined coordinate systems $\left(x_{1}, \ldots, x_{n}, t\right)$ (they are the projection of the canonical coordinates of $\mathbb{R}^{n}=$ $\left.\mathbb{R}^{n-1} \times[0,1]\right)$.

2.9. Let $\varphi \in G L(n-1, \mathbb{R}) \subset G L(n, \mathbb{R})$, where the last inclusion is induced by the inclusion $\mathbb{R}^{n-1} \hookrightarrow \mathbb{R}^{n}$ given by $\left(x_{1}, \ldots, x_{n-1}\right) \longmapsto\left(x_{1}, \ldots, x_{n-1}, t\right)$. Multiplication by $\varphi$ induces a linear map $x \mapsto \varphi x$ and we use the same letter to denote this map. Suppose that $\varphi$ is distance non-decreasing, that is $|\varphi(v)| \geq|v|, \forall v \in \mathbb{R}^{n-1}$ (or simply $|\varphi| \geq 1$ ), where the bars denote the Euclidean norm. Note that if $A=S_{t}+d t^{2}$ is a metric on $T^{n-1} \times I$ satisfying (2.1) and $\varphi$ is as before, then 
$A^{\varphi}=S_{t}^{\varphi}+d t^{2}$ (rigorously, $A_{X}^{\varphi}=\left(S_{t}\right)_{X^{\prime}}^{\varphi} \subset G L(n-1, \mathbb{R}) \subset G L(n, \mathbb{R})$, where $X^{\prime}$ and $X$ are as in (2.8)).

2.10. Lemma. Let $A$ be a Riemannian metric on $T^{n-1} \times I$ satisfying (2.1) and $f$ as in (2.3). Then given $\epsilon>0$, there is an $L$ such that, for $\alpha>L$, and $\varphi$ distance non-decreasing,

$$
-k_{0}-\epsilon<K\left(A_{\alpha}^{\varphi}\right)<-k_{1}+\epsilon .
$$

Remark. The point here is that the constant $L$ is independent of $\varphi$, provided it is distance non-decreasing.

Proof. The proof is the same as the proof of lemma 2.4 (see the appendix), except that we have to find a constant $C$, as in (a.2), that is, independent of $\varphi$ satisfying (2.9).

Let $A_{X}=\left(g_{i j}\right)$ be the representation of $A$ in the basis $X$, where $X$ is as in (2.8). Now, $A_{X}(x, t)$ is positive definite; thus, it can be written as $E^{\mathrm{T}}(x, t) \cdot E(x, t)$, for some matrix $E$ depending on $(x, t)$. Note that $E$ is smooth (and globally defined because the bundle $O(n, \mathbb{R}) \rightarrow G L(n, \mathbb{R}) \stackrel{\eta}{\rightarrow} \mathcal{P}, \eta(B)=B^{\mathrm{T}} B$, is trivial) in $(x, t)$. Because $T^{n-1} \times I$ is compact, we can find a constant $C_{1}$ such that

$$
\left|\frac{\partial^{k+s} g_{i j}}{\partial x_{i_{1}} \ldots \partial x_{i_{k}} \partial t^{s}}\right|<C_{1}, \quad\left|E_{i j}^{-1}(x, t)\right|<C_{1}, \quad \forall i, j, \quad k+s \leq 2 .
$$

For each $\left(x_{0}, t_{0}\right) \in T^{n} \times I$, define new coordinates near $\left(x_{0}, t_{0}\right)$ as follows:

$$
\begin{aligned}
& \bar{x}=E\left(x_{0}, t_{0}\right) \cdot \varphi \cdot x, \\
& \bar{t}=t
\end{aligned}
$$

where $x=\left(x_{1}, \ldots, x_{n}\right)$ are coordinates as in (2.8) (without the $t$ variable). Also, write $\bar{x}_{0}=E\left(x_{0}, t_{0}\right) \cdot \varphi \cdot x_{0}$ and $\bar{t}_{0}=t_{0}$.

Then if $\left(\bar{g}_{i j}^{\varphi}\right)$ is $A^{\varphi}$ in $(\bar{x}, \bar{t})$-coordinates (that is, $A_{\bar{X}}^{\varphi}=\left(\bar{g}_{i j}^{\varphi}\right)$ ), we have

$$
\begin{aligned}
\left(\bar{g}_{i j}^{\varphi}\right)(\bar{x}, \bar{t}) & =\left[\varphi^{-1} \cdot E^{-1}\left(x_{0}, t_{0}\right)\right]^{T} \cdot\left[\varphi^{T} \cdot A_{X}(x, t) \cdot \varphi\right] \cdot\left[\varphi^{-1} \cdot E^{-1}\left(x_{0}, t_{0}\right)\right] \\
& =E^{-T}\left(x_{0}, t_{0}\right) \cdot A_{X}(x, t) \cdot E\left(x_{0}, t_{0}\right),
\end{aligned}
$$

that is, it is independent of $\varphi$. Because $A_{X}(x, t)=E^{\mathrm{T}}(x, t) \cdot E(x, t)$, we have that $\left(\bar{g}_{i j}^{\varphi}\right)\left(\overline{x_{0}}, \overline{t_{0}}\right)$ is the identity matrix, so that (b) of (a.2) is true. To complete the proof, we have to show that (c) of (a.2) holds. By the chain rule,

$$
\left(\frac{\partial \bar{g}_{i j}^{\varphi}}{\partial \bar{x}_{1}}, \ldots, \frac{\partial \bar{g}_{i j}^{\varphi}}{\partial \bar{x}_{n}}\right)=\left[\left(\frac{\partial \bar{g}_{i j}^{\varphi}}{\partial x_{1}}, \ldots, \frac{\partial \bar{g}_{i j}^{\varphi}}{\partial x_{n}}\right)\right] \cdot\left[(\varphi)^{-1} \cdot E^{-1}\left(x_{0}, t_{0}\right)\right]
$$

and second derivatives

$$
\left(\frac{\partial^{2} \bar{g}_{i j}^{\varphi}}{\partial \bar{x}_{k} \partial \bar{x}_{l}}\right)=\left(\frac{\partial^{2} \bar{g}_{i j}^{\varphi}}{\partial x_{k} \partial x_{l}}\right) \cdot\left[(\varphi)^{-1} \cdot E^{-1}\left(x_{0}, t_{0}\right)\right]^{2} .
$$

But $\varphi$ is distance non-decreasing (i.e., $|\varphi| \geq 1$ ). Then $\varphi^{-1}$ is distance nonincreasing (i.e., $\left|\varphi^{-1}\right| \leq 1$ ), so that

$$
\begin{aligned}
\left|\left(\frac{\partial \bar{g}_{i j}^{\varphi}}{\partial \bar{x}_{1}}, \ldots, \frac{\partial \bar{g}_{i j}^{\varphi}}{\partial \bar{x}_{n}}\right)\right| & \leq\left|\varphi^{-1}\right| .\left|E^{-1}\left(x_{0}, t_{0}\right)\right| \cdot\left|\left(\frac{\partial \bar{g}_{i j}^{\varphi}}{\partial x_{1}}, \ldots, \frac{\partial \bar{g}_{i j}^{\varphi}}{\partial x_{n}}\right)\right| \\
& \leq\left|E^{-1}\left(x_{0}, t_{0}\right)\right| \cdot\left|\left(\frac{\partial \bar{g}_{i j}^{\varphi}}{\partial x_{1}}, \ldots, \frac{\partial \bar{g}_{i j}^{\varphi}}{\partial x_{n}}\right)\right|,
\end{aligned}
$$


and for the second derivatives,

$$
\begin{aligned}
\left|\left(\frac{\partial^{2} \bar{g}_{i j}^{\varphi}}{\partial \bar{x}_{k} \partial \bar{x}_{l}}\right)\right| & \leq\left|\left(\frac{\partial^{2} \bar{g}_{i j}^{\varphi}}{\partial x_{k} \partial x_{l}}\right)\right| \cdot\left|E^{-1}\left(x_{0}, t_{0}\right)\right|^{2} \cdot\left|(\varphi)^{-1}\right|^{2} \\
& \leq\left|\left(\frac{\partial^{2} \bar{g}_{i j}^{\varphi}}{\partial x_{k} \partial x_{l}}\right)\right| \cdot\left|E^{-1}\left(x_{0}, t_{0}\right)\right|^{2} .
\end{aligned}
$$

Thus we can find a constant $C$ as in (c) of (a.2) because $\left(\bar{g}_{i j}^{\varphi}\right)$ is independent of $\varphi$, and its derivatives in $\bar{x}$-coordinates can be majorated by its derivatives in $x$-coordinates, that do not depend on $\varphi$ either. This completes the proof of the lemma.

2.11. Denote by $I$ the canonical flat metric on $T^{n-1}=\mathbb{S}^{1} \times \ldots \times \mathbb{S}^{1}$. That is, $I$ written in the basis $X^{\prime}=\left\{\frac{\partial}{\partial x_{1}}, \ldots, \frac{\partial}{\partial x_{n-1}}\right\}$ (see 2.6, 2.8) is just the identity matrix.

Then, for $\varphi \in G L(n-1, \mathbb{R}), I_{X^{\prime}}^{\varphi}=\varphi^{\mathrm{T}} \varphi$ (see 2.7 ).

2.12. We say that the torus $T^{n-1}=\mathbb{S}^{1} \times \ldots \times \mathbb{S}^{1}$ with metric $I^{\varphi}$ is large, if $\left(\frac{1}{c_{n}}\right) \varphi$ is distance non-decreasing, where $c_{n}$ is a constant (that depends only on the dimension $n$ ) that will be determined on the proof of theorem 3 (see 5.1). A flat torus is large if it is isometric to $\mathbb{S}^{1} \times \ldots \times \mathbb{S}^{1}$ with metric $I^{\varphi}, I^{\varphi}$ large. Note that every flat torus is isometric to $\mathbb{R}^{n-1} / L$, with $L \subset \mathbb{R}^{n-1}$ a lattice generated by some vectors $v_{1}, \ldots, v_{n-1}$. Hence, this flat torus is isometric to $\mathbb{S}^{1} \times \ldots \times \mathbb{S}^{1}$ with metric $I^{\varphi}$, where $\varphi=\left[v_{1} \ldots v_{n-1}\right]$. Then the flat torus is large iff we can find a basis $v_{1}, \ldots, v_{n-1}$ of $L$ such that $|\varphi| \geq 1$, for $\varphi=\left[v_{1} \ldots v_{n-1}\right]$.

A cusp of the form $T^{n-1} \times[0, \infty)$ with metric $e^{-2 t} S+d t^{2}$ is large if $T^{n-1}$ with metric $S$ is large.

2.13. We say that a finite cover $p: T^{n-1} \rightarrow T^{n-1}$ corresponding to a subgroup of finite index $H$ of $\pi_{1}\left(T^{n-1}\right) \cong \mathbb{Z}^{n-1}$ is simple with respect to a basis $\mathcal{B}=\left\{v_{1}, \ldots, v_{n-1}\right\}$ of $\pi_{1}\left(T^{n-1}\right)$, if $H$ has a basis of the form $\left\{m_{1} v_{1}, \ldots, m_{n-1} v_{n-1}\right\}, m_{i} \in \mathbb{Z}$. The minimum length of the simple cover is just $\min \left\{\left|m_{i}\right|\right\}$.

2.14. Lemma. Fix a base $\mathcal{B}=\left\{v_{1}, \ldots, v_{n-1}\right\}$ of $\pi_{1}\left(T^{n-1}\right)$, and $S=I^{\varphi}$ a (flat) metric on $T^{n-1}$. Then there is a $K$ such that if $p: T^{n-1} \rightarrow T^{n-1}$ is a simple cover (with respect to $\mathcal{B}$ ) of minimum length $\geq K$, then $T^{n-1}$ with metric $p^{*} S$ is large.

Proof. Consider $T^{n-1}$ with canonical coordinates $x=\left(x_{1}, \ldots, x_{n-1}\right)$, i.e., these coordinates come from $\mathbb{R}^{n-1}$ by the covering projection $\mathbb{R}^{n-1} \rightarrow T^{n-1}$. We identify $\pi_{1}\left(T^{n-1}\right)$ with $\mathbb{Z}^{n-1} \subset \mathbb{R}^{n-1}$. Thus $v_{i} \in \mathbb{Z}^{n-1}$. Now, if $p$ is simple, then $p: T^{n} \rightarrow$ $T^{n}$ is such that $p\left(v_{i}\right)=m_{i} v_{i}$, for some $m_{i} \in \mathbb{Z}$. Let $B$ be the matrix whose columns are the $v_{i}$ 's. Then $p$ written in the basis $v_{1}, \ldots, v_{n-1}$ is given by $d=\operatorname{diag}\left\{m_{1} \ldots m_{n-1}\right\}$ and it follows that $p(x)=B d B^{-1} x$, for $x \in \mathbb{R}^{n-1}$. Consequently, the pullback of the flat metric $S=I^{\varphi}$ in $T^{n-1}$, written in the canonical basis $\left\{e_{i}\right\}$ is

$$
\left(B d B^{-1}\right)^{\mathrm{T}} \varphi^{\mathrm{T}} \varphi\left(B d B^{-1}\right)=\left(\varphi B d B^{-1}\right)^{\mathrm{T}}\left(\varphi B d B^{-1}\right) .
$$

It is now easy to see that if the length of $p$ (i.e., $\min \left\{\left|m_{i}\right|\right\}$ ) is large, we get $\left(\frac{1}{c_{n}}\right) \varphi B d B^{-1}$ distance non-decreasing. In fact, it is enough to take $K \geq$ $c_{n}|B|\left|B^{-1}\right|\left|\varphi^{-1}\right|$. This completes the proof. 


\section{Triangulation Lemmas}

Recall that if $M$ is a $P L$ manifold and $C \subset M$, a closed subset (assume $m=$ $\operatorname{dim} M \geq 6$ or $\operatorname{dim} M \geq 5$ and $\partial M \subset C)$, then there is a one-to-one correspondence between $\check{H}^{3}\left(M, C ; \mathbb{Z}_{2}\right)$ (this is Cech cohomology) and the set of concordance classes of $P L$ structures on $M$ that agree with the given one on a neighborhood of $C$. We can choose this correspondence to be such that it sends the given $P L$ structure to 0 .

Given a concordance class of triangulations $[\tau]$, by $c_{[\tau]}=c_{\tau} \in \check{H}^{3}\left(M, C ; \mathbb{Z}_{2}\right)$, we denote the corresponding cohomology class; also, given a cohomology class $c$, we write $\left[\tau_{c}\right]=[\tau]_{c}$ for the corresponding concordance class of triangulations.

We have the following

3.1. Lemma. Let $p: \tilde{M} \rightarrow M$ be a covering, $C \subset M$ closed and $m=\operatorname{dim} M \geq 6$ (or $\operatorname{dim} M \geq 5$ and $\partial M \subset C$ ). Suppose $M$ has a $P L$ structure $\tau_{0}$ and denote by $\tilde{\tau}_{0}$ the pullback $p^{*} \tau_{0}$ of $\tau_{0}$ and make these two triangulations correspond to zero in $\check{H}^{3}\left(\tilde{M}, p^{-1}(C) ; \mathbb{Z}_{2}\right)$ and $\check{H}^{3}\left(M, C ; \mathbb{Z}_{2}\right)$, respectively. Then $[\tau]_{p^{*} c}=\left[p^{*} \tau_{c}\right]$ for all $c \in \check{H}^{3}\left(M, C ; \mathbb{Z}_{2}\right)$. Equivalently, $c_{p^{*} \tau}=p^{*} c_{\tau}$ for every $P L$ structure $\tau$ on $M$.

Note that if $\tau_{1}$ and $\tau_{2}$ are concordant $P L$ structures on $M$, then $p^{*} \tau_{1}$ and $p^{*} \tau_{2}$ are also concordant.

The proof of this lemma appears in [15].

Now, given a $P L$ manifold $M$, we show how to change $P L$ structures by cutting along a closed hypersurface $N$ of $M$, and gluing back with a twist.

Denote by $M_{\chi}$ the $C A T(=P L$ or $D I F F)$ manifold obtained by cutting along $N$ (a $C A T$ closed hypersurface), and identifying by $\chi$ the two copies of $N$ so obtained, where $N$ is a $C A T$ closed hypersurface and $\chi: N \rightarrow N$ is a $C A T$ isomorphism. In what follows, we assume that the relative set is nice enough (for example, a deformation retract of a subcomplex) so that we replace Cech cohomology by singular cohomology.

3.2. Lemma. Let $M$ be a $P L$ orientable $n$-manifold, $n \geq 6, N$ a closed $P L$ hypersurface with a tubular neighborhood $g: W \cong_{P L} N \times[-1,1]$ of $N$ in $M$, where $g(N)=N \times\{0\}$, and $J \subset N$ open with $\bar{J}$ compact. Then for every $c \in H^{3}\left(M, M \backslash J ; \mathbb{Z}_{2}\right)$, there is a PL isomorphism $\chi: N \rightarrow N$, such that $M_{\chi}$ (that is, its PL structure) corresponds to $c$ (by the correspondence that sends the given $P L$ structure to 0 ) and $\chi$ is the identity outside a compact neighborhood of $\bar{J}$.

The proof of this lemma is given in [15].

3.3. Remark. Note that if $\tau$ is smoothable, then, using the differentiable $s$-cobordism theorem, we can choose $\chi$ to be smooth (see [15]).

\section{More Lemmas and Remarks}

First some remarks.

4.1. Let $M^{n}$ be hyperbolic, non-compact with finite volume and $F^{n-1} \times[0, \infty)$ one of its cusps (recall that we are identifying the cusp with $F^{n-1} \times[0, \infty)$ ). Then if $\imath: F^{n-1} \hookrightarrow F^{n-1} \times[0, \infty) \subset M$ is the inclusion, $\imath_{*}: \pi_{1}\left(F^{n-1}\right) \rightarrow \pi_{1}(M)$ is one-to-one. This is because (see 1.2 ) we can put a non-positively curved metric on 
the double $D M$ of $M$, such that $F^{n-1}$ is a totally geodesic flat submanifold of $D M$, and then the map

$$
\pi_{1}\left(F^{n-1}\right) \rightarrow \pi_{1}(M) \rightarrow \pi_{1}(D M)
$$

is one-to-one, which means that $\imath_{*}$ is one-to-one.

4.2. We claim that $T^{n-1} \times[0,3 \alpha]$ with metric $e^{-2 t} I^{\varphi}+d t^{2}$ (see 2.11) is isometric to $T^{n-1} \times[0,3]$ with metric $I_{\alpha}^{\psi}+\alpha^{2} d t^{2}$, where $\psi=\left(e^{-4 \alpha}\right) \varphi$ and the warping function is $f(\alpha, t)=e^{4 \alpha-\alpha t}$. To see this, we have $I_{\alpha}^{\psi}+\alpha^{2} d t^{2}=f(\alpha, t)^{2} I^{\left(e^{-4 \alpha}\right) \varphi}+$ $\alpha^{2} d t^{2}=e^{8 \alpha-2 \alpha t}\left[\left(e^{-4 \alpha}\right) \varphi\right]^{T}\left[\left(e^{-4 \alpha}\right) \varphi\right]+\alpha^{2} d t^{2}=\left(e^{-2 \alpha t}\right) \varphi^{T} \varphi+\alpha^{2} d t^{2}$, and note that $T^{n-1} \times[0,3]$ with metric $\left(e^{-2 \alpha t}\right) \varphi^{T} \varphi+\alpha^{2} d t^{2}$ is isometric to $T^{n-1} \times[0,3 \alpha]$ with metric $e^{-2 t} I^{\varphi}+d t^{2}$, and the isometry is simply $(x, t) \mapsto(x, \alpha t)$.

4.3. Suppose $M$ is a finite volume hyperbolic manifold of dimension $n$ and $H$ a totally geodesic hypersurface with a cusp isometric to $F^{n-2} \times[0, \infty)$ with metric $e^{-2 t} S+d t^{2}$. Then because the radius of injectivity tends to zero only in the cusps, $M$ has a cusp such that the cusp of $H$ is included in the cusp of $M$. That is, taking $x \in F^{n-2}$, then $t \mapsto(x, t) \in\{x\} \times[0, \infty)$ is a geodesic in $H$ and also in $M$, and the radius of injectivity, as a function of $t$, is strictly decreasing, so that there is a $t_{0}$ such that for $t>t_{0}$, the cusp of $H$ is contained in some cusp of $M$, and cannot leave this cusp because the radius of injectivity outside the cusps is bounded above from zero. Moreover, suppose that the cusp of $M$ is also of the form $F^{n-1} \times[0, \infty)$. Then because the only geodesics that do not leave a cusp are of the form described before (that is, $t \mapsto(x, t) \in\{x\} \times[0, \infty))$ we have that there is a flat $F^{n-2}$ inside the $F^{n-1}$ such that the inclusion of the cusps is simply this inclusion (i.e., $F^{n-2} \hookrightarrow F^{n-1}$ ) times $\left[t_{0}, \infty\right)$.

4.4. Lemma. Let $M$ be a compact differentiable $n$-manifold such that one of the components of the boundary is diffeomorphic to the $(n-1)$-torus (denote it by $\left.T^{n-1}\right)$, that is, $T^{n-1} \subset \partial M$. Suppose one of the following holds:

(i) $n$ is odd;

(ii) $n$ is even and the restriction map $H^{n-2}\left(M, \partial M \backslash T^{n-1}\right) \rightarrow H^{n-2}\left(T^{n-1}\right)$ is not onto.

Then the map $\iota_{3}: H^{3}\left(D M, D M \backslash T^{n-1}\right) \rightarrow H^{3}(D M)$ is not zero. Here $D M$ denotes the double of $M$.

Remark. We are using $\mathbb{Z}_{2}$ coefficients.

Proof. First write $D M$ as $M_{0} \cup M_{1}$, where $M_{i}=M, i=0,1$, and we are identifying the boundaries by the identity map. Denote by $p_{i}: D M \rightarrow M_{i}$ the map defined by $p_{i}(x)=x \in M_{i}$, and $\sigma: D M \rightarrow D M$, defined by $\sigma(x)=p_{i}(x)$, for $x \in M_{i+1}, i \in \mathbb{Z}_{2}$. Then $\sigma$ is an involution and $p_{i}$ is a retraction. Write also $P=T^{n-1} \times[-1,1]$ for a tubular neighborhood of $T^{n-1} \subset D M$, and assume $P \sigma$-symmetric (i.e., $\sigma P=P$ ). Let $N=D M \backslash$ int $P$. Then $N$ is contained in $M_{0} \cup M_{1} \backslash T^{n-1}$; so we consider $M_{i} \subset N$ and denote by $r_{i}: M_{i} \rightarrow N$ the inclusion.

Also, $\partial N$ is homeomorphic to two copies $T_{0}^{n-1}$ and $T_{1}^{n-1}$ of $T^{n-1}$, with $T_{i}^{n-1} \subset$ $M_{i}$. (This homeomorphism is the restriction of the tubular neighborhood projection $P \rightarrow T^{n-1}$.) Write $q_{i}: T^{n-1} \rightarrow T_{i}^{n-1} \subset \partial N, i=0,1$, for these homeomorphisms. Also, let $u_{i}: H^{*}\left(M_{i}\right) \rightarrow H^{*}\left(T^{n-1}\right)$ be the composition of the map induced by the inclusion $T_{i}^{n-1} \subset M_{i}$ with $q_{i}^{*}$. Note that $\sigma\left(T_{i}^{n-1}\right)=T_{i+1}^{n-1}, \sigma q_{i}=q_{i+1}, i \in \mathbb{Z}_{2}$ and $\left.\sigma\right|_{\partial M_{0} \backslash T^{n-1}}$ is the identity. Also, write $s_{i}: T_{i}^{n-1} \rightarrow N$ for the inclusions. Then $\sigma s_{i}=s_{i+1} \sigma, i \in \mathbb{Z}_{2}$. 
Define $\phi_{*}: H^{*}(N) \rightarrow H^{*}\left(T^{n-1}\right)$ by $\phi=q_{0}^{*} s_{0}^{*}+q_{1}^{*} s_{1}^{*}$. It is not difficult to see that the following diagram is commutative:

$$
\begin{array}{ccc}
H^{k}(N) & \cong & H^{k}\left(D M \backslash T^{n-1}\right) \\
\phi \downarrow & & \downarrow \delta \\
H^{k}\left(T^{n-1}\right) & \cong & H^{k+1}\left(D M, D M \backslash T^{n-1}\right)
\end{array}
$$

where the lower isomorphism is excision composed with the canonical isomorphism $H^{k}\left(T^{n-1}\right) \rightarrow H^{k+1}\left(T^{n-1} \times[0,1], \partial\right)$. Let $\epsilon: H^{*}\left(M_{0}, \partial M_{0} \backslash T^{n-1}\right) \rightarrow H^{*}(N)$ be the composition of the excision map $H^{*}\left(M_{0}, \partial M_{0} \backslash T^{n-1}\right) \rightarrow H^{*}\left(N, M_{1}\right)$ with the inclusion $H^{*}\left(N, M_{1}\right) \rightarrow H^{*}(N)$. Let $\eta: H^{*}\left(M_{0}, \partial M_{0} \backslash T^{n-1}\right) \rightarrow H^{*}\left(M_{0}\right)$ be the inclusion. We have the commutative diagram

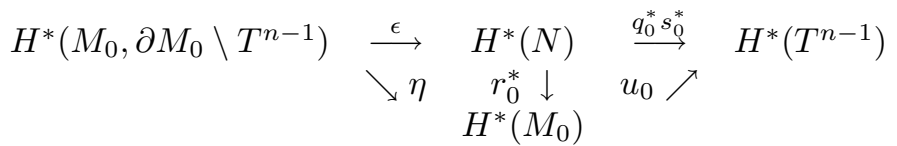

Define $\rho=q_{0}^{*} s_{0}^{*} \epsilon$. Note that this $\rho$ is the map that appears in $(i i)$ of the statement of the lemma.

Consider now the following exact sequence:

$$
\rightarrow H^{k-1}(D M) \rightarrow H^{k-1}\left(D M \backslash T^{n-1}\right) \stackrel{\delta}{\rightarrow} H^{k}\left(D M, D M \backslash T^{n-1}\right) \stackrel{\iota_{k}}{\rightarrow} H^{k}(D M) \rightarrow .
$$

Claim. $\iota_{k}=0$ implies $\rho_{k-1}$ onto.

Proof of the claim. $\iota_{k}=0$ implies $\delta$ onto, which in turn implies $\phi_{k-1}$ onto. Hence, given $c \in H^{k-1}\left(T^{n-1}\right)$, there is $b \in H^{k-1}(N)$, with $\phi_{k-1} b=c$ (we just write $\phi b=c)$. Let $d=b-\sigma^{*} b \in H^{k-1}(N)$, and $a=r_{0}^{*} d=\left.d\right|_{M_{0}}$. Note that $\left.a\right|_{\partial M_{0} \backslash T^{n-1}}=$ $\left.\left.d\right|_{M_{0}}\right|_{\partial M_{0} \backslash T^{n-1}}=\left.d\right|_{\partial M_{0} \backslash T^{n-1}}=\left.\left(b-\sigma^{*} b\right)\right|_{\partial M_{0} \backslash T^{n-1}}=\left.b\right|_{\partial M_{0} \backslash T^{n-1}}-\sigma^{*}\left(\left.b\right|_{\partial M_{0} \backslash T^{n-1}}\right)$ $=0$ because $\left.\sigma\right|_{\partial M_{0} \backslash T^{n-1}}=I d$. Hence there is $e \in H^{k-1}\left(M_{0}, \partial M_{0} \backslash T^{n-1}\right)$ with $a=\eta e$.

Then, we get $\rho e=q_{0}^{*} s_{0}^{*} \epsilon e=u_{0} \eta e=u_{0} a=u_{0} r_{0}^{*} d=q_{0}^{*} s_{0}^{*} d=q_{0}^{*} s_{0}^{*}\left(b-\sigma^{*} b\right)=$ $q_{0}^{*} s_{0}^{*} b-q_{0}^{*} s_{0}^{*} \sigma^{*} b=q_{0}^{*} s_{0}^{*} b-q_{0}^{*} \sigma^{*} s_{1}^{*} b=q_{0}^{*} s_{0}^{*} b-q_{1}^{*} s_{1}^{*} b=q_{0}^{*} s_{0}^{*} b+q_{1}^{*} s_{1}^{*} b=\phi b=c$. (Recall that we are using $\mathbb{Z}_{2}$ coefficients.) This proves the claim.

Consequently, if $\iota_{3}=0$, then $\rho_{2}$ is onto, so that $\rho_{4}$ is also onto (this is because $\rho$ is a ring homomorphism and also because $H^{2}\left(T^{n-1}\right) \otimes H^{2}\left(T^{n-1}\right) \rightarrow H^{4}\left(T^{n-1}\right)$, given by the cup product, is an epimorphism). Repeating this process we obtain that $\rho_{2 k}$ is onto. Now if $n$ is odd, this means that $\rho_{n-1}$ is onto. This is a contradiction, because $\rho_{n-1}=0$ and $H^{n-1}\left(T^{n-1}\right)$ is not zero. If $n$ is even, then $\rho_{n-2}$ is onto, which contradicts $(i i)$ of the statement of the lemma. This completes the proof.

4.5. Suppose that we have a totally geodesic hypersurface $H$ with a cusp of the form $T^{n-2} \times[0, \infty)$ contained in a cusp $C$ of the manifold $M$, also of the form $T^{n-1} \times[0, \infty)$. Suppose also that this is the only cusp of $H$ contained in the cusp $C$ of $M$ (this last statement makes sense; see 4.3). Then (ii) of lemma (4.4) holds for $M$. To prove this, let $x \in H^{n-2}\left(T^{n-1}\right)$ be the cohomology class dual to a loop $\gamma \subset T^{n-1} \subset C$ that intersects $T^{n-2} \subset T^{n-1}$ transversally in one point (see 4.8). Let also $y \in H^{n-2}\left(\partial M, \partial M \backslash T^{n-1}\right)$ be the image of $x$ under the excision isomorphism $H^{n-2}\left(T^{n-1}\right) \cong H^{n-2}\left(\partial M, \partial M \backslash T^{n-1}\right)$. Then $y$ is the restriction of a class in $H^{n-2}\left(M, \partial M \backslash T^{n-1}\right)$ if and only if $\delta^{\prime}(y)=0$, where $\delta^{\prime}: H^{n-2}\left(\partial M, \partial M \backslash T^{n-1}\right) \rightarrow$ $H^{n-1}(M, \partial M)$ denotes the connecting homomorphism in the exact sequence of the 
triple $\left(M, \partial M, \partial M \backslash T^{n-1}\right)$. Since $\gamma$ intersects $H$ transversally in one point, $\delta^{\prime}(y)$ evaluates to 1 on the fundamental class of the hypersurface $H$. Consequently, $\delta^{\prime}(y) \neq 0$, and $x$ is not the restriction of a class in $H^{n-2}\left(\partial M, \partial M \backslash T^{n-1}\right)$.

We will need some results about involutions on a flat torus.

4.6. Lemma. Let $\iota \neq 1$ be an isometric involution on a flat torus $T^{n}$. Suppose that there is a (connected) totally geodesic flat $(n-1)$-torus $N$ contained in the fixed point set fix $(\iota)$ of $\iota$. Then, either

(i) $N=$ fix $(\iota)$, or

(ii) there is an isometry $\eta: T^{n} \rightarrow \mathbb{S}_{b}^{1} \times N$, for some $b>0$, such that $\eta \iota \eta^{-1}(z, x)$ $=(\bar{z}, x)$.

Here $\mathbb{S}_{b}^{1}=\{z \in \mathbb{C}:|z|=b\}$ and the bar denotes conjugation.

Proof. Let $L \subset \mathbb{R}^{n}$ be a lattice generated by $v_{1}, \ldots, v_{n} \in \mathbb{R}^{n}$ such that $\mathbb{R}^{n} / L \cong_{I S O}$ $T^{n}\left(\mathbb{R}^{n}\right.$ with its standard metric) and such that $\pi(0) \in N$, where $\pi: \mathbb{R}^{n} \rightarrow$ $\mathbb{R}^{n} / L \cong{ }_{I S O} T^{n}$ is the covering projection.

Let $\tilde{\iota}: \mathbb{R}^{n} \rightarrow \mathbb{R}^{n}$ be a lifting of $\iota$, such that $\tilde{\iota}(0)=0$. Let $H$ be the component of $\pi^{-1}(N)$ that contains 0 . Then $H$ is an $(n-1)$-linear subspace of $\mathbb{R}^{n}$ and $\left.\tilde{\iota}\right|_{H}=I d$. Hence $\tilde{\iota}$ is a Euclidean reflection, i.e., $\tilde{\iota}(x)=x-2\langle x, v\rangle v$, where $v$ is orthonormal to $H$.

We can suppose (after a rotation) that $H=\{0\} \times \mathbb{R}^{n-1} \subset \mathbb{R}^{n}$ or, equivalently, that $v=e_{1}=(1,0, \ldots, 0)$. Let $a=\min \left\{t>0: t e_{1}+H \subset L+H\right\}$. Then $a>0$ and $a e_{1}+H \subset L+H$.

Let $p: \mathbb{R}^{n} \rightarrow \mathbb{R}$ be the projection to the first coordinate $x \mapsto\left\langle e_{1}, x\right\rangle$.

Claim 1. a generates the subgroup $p(L)$ of $\mathbb{R}$.

To see this, note that $t \in p(L)$ iff $t e_{1}+H \subset L+H$, and because $p(L)$ is a discrete subgroup of $\mathbb{R}$, then it is generated by $\min \left\{t>0: t e_{1}+H \subset L+H\right\}=a$.

Claim 2. $2 a e_{1} \in L$.

Because $\left.\iota\right|_{N}=I d$ and $\pi\left(a e_{1}\right) \in \pi(L+H)=\pi(H)=N$, we get $-a e_{1}=\tilde{\iota}\left(a e_{1}\right)=$ $a e_{1}+u$, for some $u \in L$. Hence $2 a e_{1}=-u \in L$. This proves claim 2 .

By claims 1 and $2, L \cap \mathbb{R} e_{1}$ is generated either by $2 a e_{1}$ or $a e_{1}$.

Claim 3. $\pi^{-1}(f i x(\iota))=\bigcup\left\{s e_{1}+H: 2 s e_{1} \in L\right\}$.

Given $x \in \mathbb{R}^{n}$, write $x=s e_{1}+y, y \in\{0\} \times \mathbb{R}^{n-1}$. Then $x \in \pi^{-1}($ fix $(\iota))$ iff $2 s e_{1}=x-\tilde{\iota}(x) \in L$. The claim follows.

To finish the proof we have two cases.

First case. $a e_{1} \notin L$.

In this case, $2 a e_{1}$ generates $L \cap \mathbb{R} e_{1}$. Thus $2 s e_{1} \in L$ iff $s=m a$, for some $m \in \mathbb{Z}$. Hence $\pi^{-1}(f i x(\iota))=\bigcup_{m \in \mathbb{Z}} m_{a e_{1}}+H$ and we get $f i x(\iota)=\pi\left(\pi^{-1}(\right.$ fix $\left.(\iota))\right)=$ $\pi\left(\bigcup_{m \in \mathbb{Z}}\right.$ mae $\left._{1}+H\right) \subset \pi(L+H)=\pi(H)=N$. Consequently, $N=f i x(\iota)$, and this is $(i)$ of the statement of the theorem.

Second case. $a e_{1} \in L$.

In this case, $a e_{1}$ generates $L \cap \mathbb{R} e_{1}$. Thus $2 s e_{1} \in L$ iff $s=\frac{1}{2} m a$, for some $m \in \mathbb{Z}$. Hence $\pi^{-1}($ fix $(\iota))=\bigcup_{m \in \mathbb{Z}} \frac{1}{2} m a+H$ and we get $f i x(\iota)=\pi\left(\pi^{-1}(\right.$ fix $\left.(\iota))\right)=$ $\pi\left(\bigcup_{m \in \mathbb{Z}} \frac{1}{2} m a+H\right)=\pi(H) \cup \pi\left(\frac{1}{2} a e_{1}+H\right)=N+N^{\prime}$, where $N^{\prime}=\pi\left(\frac{1}{2} a e_{1}+H\right)$ is disjoint from $N$. 
Now, because $a$ is minimum, each $v_{i}$ can be written as $v_{i}=m_{i} a e_{1}+y_{i}, y_{i} \in H$, $m_{i} \in \mathbb{Z}$. But $a e_{1} \in L$ implies $y_{i} \in L$. Hence we have that $L=\mathbb{Z} a e_{1} \oplus(L \cap H)$. Then $\pi_{1} T^{n} \cong \pi_{1} N \oplus \mathbb{Z}$, where $\mathbb{Z}$ is generated by the loop $\pi\left(\left\{t e_{1}: 0 \leq t \leq 1\right\}\right)$, orthogonal to $N$. The lemma follows.

Remarks. 1. If $a e_{1} \notin L$, that is, if $N=f i x(\iota)$, we say that $N$ is a double $(n-1)$ subtorus of $T^{n-1}$, or, that $\iota$ is a double isometric involution. If $a e_{1} \in L$, that is, if (ii) of the statement of the lemma above holds, we say that $N$ is a simple $(n-1)$-subtorus of $T^{n-1}$, or, that $\iota$ is a simple isometric involution.

2. Note that if $\iota$ is simple, then $\pi_{1} T^{n} \cong \mathbb{Z} e \oplus L_{1}$, where $\left.\iota_{*}\right|_{L_{1}}=I d$ and $\iota_{*} e=-e$. Here $\iota_{*}: \pi_{1} T^{n} \rightarrow \pi_{1} T^{n}$ is the induced homomorphism. The next corollary proves the converse.

4.7. Corollary. Let $\iota: T^{n} \rightarrow T^{n}$ be an isometric involution such that $\pi_{1} T^{n} \cong$ $\mathbb{Z} e \oplus L_{1}$, with $\left.\iota_{*}\right|_{L_{1}}=I d$ and $\iota_{*} e=-e$. Then $\iota$ is simple.

Proof. We use the notation of the proof of lemma 4.6. Hence, we identify $\pi_{1} T^{n}$ with the lattice $L$ and $L_{1}$ with $L \cap H$.

If $a e_{1} \in L$, we are done, by definition of simple. Suppose $a e_{1} \notin L$. Then, we should have $L=\mathbb{Z}\left(2 a e_{1}\right) \oplus(L \cap H)$. But $a e_{1}+H \subset L+H=\left[\mathbb{Z}\left(2 a e_{1}\right)+(L \cap H)\right]+H=$ $\mathbb{Z}\left(2 a e_{1}\right)+H$. Hence $a e_{1}=2 m^{2} e_{1}+h$, for some $h \in H, m \in \mathbb{Z}$. This is a contradiction because $\left\langle e_{1}, h\right\rangle=0$ and $a \neq 0$.

4.8. Lemma. Let $T_{i}, i=1,2,3$, be three mutually orthogonal flat $(n-1)$-subtori of a flat $n$-torus $T^{n}$, such that, for each $i, T^{n}$ is isometric to $T_{i} \times \mathbb{S}_{b_{i}}^{1}$, for some $b_{i}>0$. Then the flat codimension-three totally geodesic submanifold $N=\bigcap_{i=1}^{3} T_{i}$ is connected.

Proof. Let $\pi: \mathbb{R}^{n} \rightarrow T^{n}$ be the covering projection, where we consider $\mathbb{R}^{n}$ with its canonical metric. We can assume, after a rotation, that $\pi\left(P_{i}\right)=T_{i}, i=1,2,3$, where $P_{i}=\left\{x_{i}=0\right\}$. Let $L$ be a lattice such that $\mathbb{R}^{n} / L \cong \cong_{I S O} T^{n}$. Hence $L=\mathbb{Z} e_{1} \oplus \mathbb{Z} e_{2} \oplus \mathbb{Z} e_{3} \oplus\left(L \cap P_{1} \cap P_{2} \cap P_{3}\right)$ and $N=\pi\left(\left\{x_{1}=x_{2}=x_{3}=0\right\}\right)$. The result follows.

4.9. Lemma. Let $N^{k}$ be a totally geodesic compact $k$-submanifold of a flat $n$-torus $T^{n}$. Then there is a totally geodesic compact $(n-k)$-submanifold intersecting $N$ transversally in one point.

Proof. Let $L \subset \mathbb{R}^{n}$ be a lattice generated by $v_{1}, \ldots, v_{n} \in \mathbb{R}^{n}$ such that $\mathbb{R}^{n} / L \cong_{I S O}$ $T^{n}\left(\mathbb{R}^{n}\right.$ with its standard metric) and such that $\pi(0) \in N$, where $\pi: \mathbb{R}^{n} \rightarrow$ $\mathbb{R}^{n} / L \cong_{I S O} T^{n}$ is the covering projection.

Let $H=\pi^{-1}(N)$. Then $H$ is a $k$-linear subspace of $\mathbb{R}^{n}$ and, after a rotation, we can suppose $H=\{0\} \times \mathbb{R}^{k} \subset \mathbb{R}^{n-k} \times \mathbb{R}^{k}=\mathbb{R}^{n}$. Let $p: \mathbb{R}^{n} \rightarrow \mathbb{R}^{n-k}$ be the projection. Then $p(L)$ is an abelian discrete cocompact subgroup of $\mathbb{R}^{n-k}$, that is, it is a lattice generated by some $a_{1}, \ldots, a_{n-k}$. Let $v_{i} \in L$ be such that $p\left(v_{i}\right)=a_{i}$, $i=1, \ldots, n-k$.

Let $(b, y) \in L, b \in \mathbb{R}^{n-k}, y \in \mathbb{R}^{k}$. We write $v_{i}=\left(a_{i}, y_{i}\right)$, for some $y_{i} \in \mathbb{R}^{k}$. Because the $a_{i}$ 's generate $p(L), b=\sum m_{i} a_{i}, m_{i} \in \mathbb{Z}$. Hence,

$$
(b, y)=\sum m_{i} v_{i}+\left[(b, y)-\sum m_{i} v_{i}\right]=\sum m_{i} v_{i}+\left(0, y-\sum m_{i} y_{i}\right),
$$


which implies that $\left(0, y-\sum m_{i} y_{i}\right) \in L \cap \mathbb{R}^{k}$. This proves that $L=\left(L \cap \mathbb{R}^{k}\right) \oplus L_{1}$, where $L_{1} \subset L$ is the lattice of $\mathbb{R}^{n-k}$ generated by $v_{1}, \ldots, v_{n-k}$. Let $J$ be the $(n-k)$ subspace generated by the $v_{i}$ 's. Then it is easy to see that the compact totally geodesic $(n-k)$-submanifold $P=\pi(J)$ satisfies the conditions of the lemma.

Finally, we will need the following result about geodesic spaces (see [6] for definitions).

4.10. Lemma. Let $X$ be a compact non-positively curved geodesic space. Then there exists an abelian subgroup of rank $k$ in $\pi_{1}(X, *)$ if and only if there is a flat $k$-torus immersed isometrically and totally geodesically in $X$. If $X$ is a $P L$ space with a piecewise flat metric, the immersion is also PL.

Proof. The first part is the well-known flat torus theorem for geodesic spaces (see 6]). To complete the proof of the theorem, assume that $X$ is piecewise flat, and we have to prove that the immersion of the torus $T^{k}$ in $X$ is $P L$, but this follows from the next trivial lemma.

4.11. Lemma. Let $g: A \rightarrow K$ be an isometric and totally geodesic embedding of piecewise flat finite simplicial complexes. Then $g$ is $P L$.

Proof. Totally geodesic isometries send geodesics to geodesics linearly (in fact, by translations). Hence the lemma follows by definition (use the definition given in [16]).

\section{Proof of Theorem 3}

Theorem 3. Let $M$ be a non-compact finite volume hyperbolic $n$-manifold, $n>5$, with a large cusp, $C_{M}$, diffeomorphic to $T^{n-1} \times(0, \infty)$. Assume either

(1) $n$ is odd, or

(2) $n$ is even and there is a totally geodesic hypersurface $N \subset M$, with exactly one cusp contained in the cusp $C_{M}$ of $M$.

Then the double DM of $M$ admits (at least) two different (smoothable) $P L$ structures admitting Riemannian metrics with non-positive curvature.

Proof. Consider $T^{n-1} \times(0,3)$ with canonical flat metric $I+d t^{2}$ (see 2.11). By the triangulation lemma 3.2 for every element $c=c_{\tau} \in H^{3}\left(T^{n-1} \times(0,3), T^{n-1} \times\right.$ $\left.(0,3) \backslash T^{n-1} \times\{2\}\right) \cong H^{2}\left(T^{n-1}\right)$, there is a $P L$ homeomorphism $\chi_{\tau}$ such that if we glue $T^{n-1} \times(0,2]$ with $T^{n-1} \times[2,3)$ by identifying $(x, 2) \in T^{n-1} \times(0,2]$ with $\left(\chi_{\tau}(x), 2\right) \in T^{n-1} \times[2,3)$ we obtain a triangulation non-concordant (modulo the complement of $T^{n-1} \times\{2\}$ ) with the canonical one (that is, the one induced by the flat structure). We know that all these triangulations are smoothable (see [17] p. 227) so that we suppose all the $\chi_{\tau}$ differentiable (see 3.3).

Because the set of metrics is convex, for each $\tau \in H^{3}\left(T^{n-1} \times(0,3), T^{n-1} \times(0,3) \backslash\right.$ $\left.T^{n-1} \times\{2\}\right)$ we can choose a metric $B_{\tau}$ on $T^{n-1} \times[1,2]$ satisfying $(2.1)$ and also the following:

(a) $\left.B_{\tau}\right|_{T^{n-1} \times\{1\}}=\left.I\right|_{T^{n-1} \times\{1\}}$,

(b) $\left.B_{\tau}\right|_{T^{n-1} \times\{2\}}=\left.\chi_{\tau}^{*} I\right|_{T^{n-1} \times\{2\}}$, where $\chi=\chi_{\tau}$,

(c) $\left.B_{\tau}\right|_{T^{n} \times\{t\}}$ is constant near $t=1,2$.

Define now a metric $A_{\tau}$ on $\left(T^{n-1} \times(0,3), \tau\right)$ by

$$
A_{\tau}= \begin{cases}B_{\tau} & t \in[1,2], \\ I & t \in(0,1] \cup[2,3) .\end{cases}
$$


We apply now lemma (2.10) to each $A_{\tau}, \epsilon<1$, and $f(\alpha, t)=e^{4 \alpha-t}$ (note that this $f$ satisfies $(2.3)$ for $t \in[0,3]$ with $\left.-\ell_{1}=\ell_{2}=1\right)$. Let $L$ be the maximum over all elements $c_{\tau} \in H^{3}\left(T^{n-1} \times(0,3), T^{n-1} \times(0,3) \backslash T^{n-1} \times\{2\}\right)$ of the constants we get from lemma (2.10). This means that the metrics $\left(A_{\tau}\right)_{\alpha}^{\varphi}$ are non-positively curved for $\alpha>L$ and that $\varphi$ is distance non-decreasing (this is because $-\ell_{1}=\ell_{2}=1$ ) and $\epsilon<1$. By taking $\epsilon$ small, we can have curvature as close to -1 as we want.

5.1. Now define $c_{n}=e^{4(L+1)}$.

We now try to fit $T^{n-1} \times(0,3)$ with these non-positively curved exotic triangulations on the double $D M$ of $M$. Recall that we make no distinction between the cusp and $T^{n} \times[0, \infty)$.

Let $M$ be as in the statement of the theorem and also let one of the cusps be isometric to $T^{n-1} \times[0, \infty)$ with metric $e^{-2 t} I^{\varphi}+d t^{2}$. Assume that the cusp is large. That means that $\left(\frac{1}{c_{n}}\right) \varphi=\left(\frac{1}{e^{(4(L+1)}}\right) \varphi$ is distance non-decreasing. This implies that $\left(A_{\tau}\right)_{L+1}^{\left(\frac{1}{(e 4(L+1)}\right) \varphi}$ is non-positively curved.

Consider the inclusion $T^{n-1}=T^{n-1} \times\{2 \alpha\} \subset T^{n-1} \times[\alpha, 2 \alpha] \subset M \subset D M$ (to be able to double $M$, we are cutting the cusp far away from $T^{n-1} \times[\alpha, 2 \alpha]$, that is, choose $b>2 \alpha$ in section 1 ). Then $D M_{\chi_{\tau}}$ (obtained by cutting along $T^{n-1} \times\{2 \alpha\}$ and gluing back with $\chi_{\tau}$ ) admits a non-positively curved metric: define it to be the old one (the hyperbolic metric) outside $T^{n-1} \times[\alpha, 2 \alpha]$ and inside $T^{n-1} \times[\alpha, 2 \alpha]$ to be the pullback of $\left(A_{\tau}\right)_{L+1}^{\left(\frac{1}{\left(e^{4(L+1)}\right) \varphi}\right.}$ by the diffeomorphism $T^{n-1} \times(0,3 \alpha) \rightarrow T^{n-1} \times(0,3),(x, t) \longmapsto\left(x, \frac{t}{\alpha}\right)$. Note that this metric is well defined because $\left(A_{\tau}\right)_{L+1}^{\frac{(1}{\left(e^{4(L+1)}\right) \varphi}}$ coincides with $I_{L+1}^{\left(\frac{1}{\left(e^{4(L+1)}\right) \varphi}\right.}+\alpha^{2} d t^{2}$ for $t \in(0,1] \cup[2,3)$ and $T^{n-1} \times(0,3)$ with this last metric is isometric to $T^{n-1} \times(0,3 \alpha)$ with metric $e^{-2 t} I^{\varphi}+d t^{2}($ see 4.2$)$.

Thus, doubling $M$, for each $\tau \in H^{3}\left(T^{n-1} \times(0,3), T^{n-1} \times(0,3) \backslash T^{n-1} \times\{2\}\right)$, we obtain in this way non-positively curved triangulations non-concordant to the canonical one modulo the complement of $T^{n-1} \times\{2 \alpha\}$.

Now, if $n$ is odd, lemma 4.4(i) tells us that at least one of these $\tau$ 's is nonconcordant to the canonical triangulation. If $n$ is even, then condition (2) of the theorem together with remark 4.5 imply again that at least one of these $\tau$ 's is non-concordant to the canonical triangulation, and corollary 9.6 implies that this non-concordant $P L$ structure is, in fact, not equivalent to the canonical one. This completes the proof of theorem 3.

\section{Proof of Theorem 4}

Here we prove theorem 4 .

Theorem 4. Let $M, N$ be two non-compact finite volume real hyperbolic manifolds such that

(1) $N$ is a totally geodesic submanifold of $M$,

(2) $\operatorname{dim} M=n \geq 5, \operatorname{dim} N=n-3$,

(3) the normal bundle of $N$ is trivial,

(4) $M$ has a cusp $C_{M}$ diffeomorphic to $T^{n-1} \times(0, \infty)$,

(5) $N$ has exactly one cusp contained in $C_{M}$.

Then the double DM of $M$ has a differentiable structure $\mathcal{E}$, such that

(i) $(D M, \mathcal{E})$ is not $P L$ equivalent to $D M$, and 
(ii) $(D M, \mathcal{E})$ does not admit a Riemannian metric with non-positive curvature.

In fact, DM does not even admit a piecewise flat metric with non-positive curvature.

Proof. Note first that $D N \subset D M$ also has trivial normal bundle and we denote it by $\mathbb{D}^{3} \times D N$. Note also that the cross section of $C_{M}$ is diffeomorphic to $T^{n-1}$, and we just write $T^{n-1} \subset C_{M} \subset M \subset D M$, and because $N$ is totally geodesic in $M$, $D N \cap T^{n-1}=N \cap T^{n-1}$ is a flat $(n-4)$-torus that we denote by $T^{n-4}$.

Recall that the set of concordance classes of $P L$ structures on $D M$ is in one-toone correspondence with $H^{3}\left(D M, \mathbb{Z}_{2}\right)$ and assume that the $P L$ structure induced by the hyperbolic structure on $M$ corresponds to $0 \in H^{3}\left(D M, \mathbb{Z}_{2}\right)$. Recall also that $H^{3}\left(D M, \mathbb{Z}_{2}\right) \cong[D M, T O P / P L]$, the set of homotopy classes of maps from $D M$ to $T O P / P L$.

Consider the map

$D M \rightarrow D M /\left[D M \backslash\left(\mathbb{D}^{3} \times D N\right)\right] \cong \mathbb{D}^{3} \times D N / \partial\left(\mathbb{D}^{3} \times D N\right) \rightarrow \mathbb{D}^{3} / \partial \mathbb{D}^{3} \stackrel{\alpha}{\rightarrow} T O P / P L$

where the first maps are collapsing maps and $\alpha: \mathbb{D}^{3} / \partial \mathbb{D}^{3} \rightarrow T O P / P L$ is the generator of $\pi_{3}(T O P / P L) \cong \mathbb{Z}_{2}$. Call this map $\theta: D M \rightarrow T O P / P L$. Then $\theta$ corresponds to the cohomology class $c \in H^{3}\left(D M, \mathbb{Z}_{2}\right)$ dual to the homology class determined by $D N$, and this determines a $P L$ structure $\Sigma$ on $D M . \Sigma$ is not concordant to the canonical $P L$ structure on $D M$ because the homology class determined by $D N$ is not zero ( $D N$ intersects transversally a three torus $T^{3} \subset T^{n-1}$ in one point; see 4.8). Because $\pi_{3}(T O P / P L) \cong \pi_{3}(T O P / O), \Sigma$ is smoothable, so that it is induced by a differentiable structure $\mathcal{E}$ on $D M$. Also, because of corollary $9.6, \Sigma$ (or $\mathcal{E}$ ) is not $P L$ equivalent to the canonical $P L$ structure.

Consider now the covering corresponding to $\pi_{1}\left(T^{n-1}\right) \subset \pi_{1}(D M)$. This covering is diffeomorphic to $T^{n-1} \times \mathbb{R}$. Denote by $p: T^{n-1} \times \mathbb{R} \rightarrow D M$ the covering projection. Because of lemma $3.1, p^{*} \Sigma$ corresponds to $p^{*} c$.

Claim. $p^{*} c=\operatorname{dual}\left(T^{n-4} \times \mathbb{R}\right) \in H^{3}\left(T^{n-1} \times \mathbb{R}, \mathbb{Z}_{2}\right)$.

Proof of the claim. $T^{n-1} \subset M \subset D M$ has a normal neighborhood $U \cong T^{n-1} \times$ $[0,1]$, and note that $U \cap D N \cong T^{n-4} \times[0,1]$. This normal neighborhood lifts to a normal neighborhood $U^{\prime}, U^{\prime} \subset T^{n-1} \times \mathbb{R}$, such that $\left.p\right|_{U^{\prime}} U^{\prime} \rightarrow U$ is a homeomorphism and it sends $p^{-1}(D N) \cap U^{\prime}$ homeomorphically onto $D N \cap U \cong$ $T^{n-4} \times[0,1]$. Then $p^{-1}(D N) \cap U^{\prime}=\left(T^{n-4} \times \mathbb{R}\right) \cap U^{\prime}$, but the restriction map $H^{3}\left(T^{n-1} \times \mathbb{R}\right) \rightarrow H^{3}\left(U^{\prime}\right)$ induced by the inclusion is an isomorphism. The claim follows.

Let $\mathcal{T}^{n-1}$ be the $P L$ exotic (differentiable) $(n-1)$-torus whose $P L$ structure corresponds to the cohomology class dual to $T^{n-4} \subset T^{n-1}$. Then the claim implies

$$
\left(T^{n-1} \times \mathbb{R}, p^{*} \mathcal{E}\right) \cong_{P L} \mathcal{T}^{n-1} \times \mathbb{R},
$$

and by choosing the right differentiable structure on $\mathcal{T}^{n-1}$, we even have

$$
\left(T^{n-1} \times \mathbb{R}, p^{*} \mathcal{E}\right) \cong{ }_{D I F F} \mathcal{T}^{n-1} \times \mathbb{R} .
$$

Suppose now that $(D M, \mathcal{E})$ admits a non-positively curved Riemannian metric. Then, by [13, there is an isometric (differentiable) embedding $j: T^{n-1} \rightarrow(D M, \mathcal{E})$ $\left(T^{n-1}\right.$ here has its canonical differentiable structure) such that $j_{*}\left(\pi_{1}\left(T^{n-1}\right)\right)=$ $\pi_{1}\left(T^{n-1}\right) \subset \pi_{1}(D M)$. Then $j$ lifts to an embedding $j^{\prime}: T^{n-1} \rightarrow\left(T^{n-1} \times\right.$ 
$\left.\mathbb{R}, p^{*} \mathcal{E}\right) \cong_{D I F F} \mathcal{T}^{n-1} \times \mathbb{R}$. But this implies that $T^{n-1}$ and $\mathcal{T}^{n-1}$ are differentiably $h$-cobordant. Hence $T^{n-1} \cong_{D I F F} \mathcal{T}^{n-1}$ (because $W h\left(\pi_{1}\left(T^{n-1}\right)\right)=0$ ), a contradiction.

To see that $(D M, \mathcal{E})$ does not even admit a piecewise flat non-positively curved metric, use lemma 4.9 and proceed in the same way. That is, suppose now that $(D M, \mathcal{E})$ admits a piecewise flat non-positively curved metric. Then, by lemma 4.9 , there is an isometric $P L$ embedding $j: T^{n-1} \rightarrow(D M, \mathcal{E})\left(T^{n-1}\right.$ here has its canonical $P L$ structure) such that $j_{*}\left(\pi_{1}\left(T^{n-1}\right)\right)=\pi_{1}\left(T^{n-1}\right) \subset \pi_{1}(D M)$. Then $j$ lifts to an embedding $j^{\prime}: T^{n-1} \rightarrow\left(T^{n-1} \times \mathbb{R}, p^{*} \mathcal{E}\right) \cong_{D I F F} \mathcal{T}^{n-1} \times \mathbb{R}$. But this implies that $T^{n-1}$ and $\mathcal{T}^{n-1}$ are $P L h$-cobordant. Hence $T^{n-1} \cong_{P L} \mathcal{T}^{n-1}$ (because $\left.W h\left(\pi_{1}\left(T^{n-1}\right)\right)=0\right)$, a contradiction. This proves theorem 4 .

\section{The Manifolds $M(\mathcal{Q}, a)$ and Proof of Theorem 5}

Let $\mathcal{Q}$ be a quadratic form of signature $(n, 1)$ with rational coefficients, $O(\mathcal{Q})$, the group of $n+1$ square matrices with real entries that preserve $\mathcal{Q}$ and $O(\mathcal{Q})_{\mathbb{Z}}=$ $O(\mathcal{Q}) \cap G L(n+1, \mathbb{Z})$. Let $C \in G L(n+1, \mathbb{R})$ be such that $C^{\mathrm{T}} \mathcal{Q} C=\operatorname{diag}\{1, \ldots, 1,-1\}$. Hence $C^{-1}\left(O(\mathcal{Q})_{\mathbb{Z}}\right) C \subset C^{-1} O(\mathcal{Q}) C \subset O(n, 1 ; \mathbb{R}) . O(\mathcal{Q}) / O(\mathcal{Q})_{\mathbb{Z}}$ has finite volume (see [5]). In general, $O(\mathcal{Q})_{\mathbb{Z}}$ is not torsion free, but it has finite index subgroups that are torsion free. These subgroups are the principal congruence subgroups defined in the following way. Let $\mathcal{R}$ be a commutative ring with unity, $\mathcal{I}$ an ideal of $\mathcal{R}$ such that $\mathcal{R} / \mathcal{I}$ is finite and $\Gamma$ a group of square $n$ by $n$ matrices with entries in $\mathcal{R}$. Then the congruence subgroup $\Gamma_{\mathcal{I}}$ is the subgroup of $\Gamma$ of all the matrices congruent to the identity modulo $\mathcal{I}$. Then $\Gamma_{\mathcal{I}}$ is of finite index. In our case, if we take $\mathbb{Z}$ (or any ideal of it) as being our ring $\mathcal{R}$ and $\Gamma$ as $O(Q)_{\mathbb{Z}}$, it is known that (see [4]) for all but finite prime ideals $\mathcal{P}, \Gamma_{\mathcal{P}}$ is torsion free, so that $C^{-1} \Gamma_{\mathcal{P}} C \backslash O(n+1,1 ; \mathbb{R}) / O(n, \mathbb{R})$ is a complete finite volume hyperbolic manifold. Then for the integers $a$, such that $\left(O(\mathcal{Q})_{\mathbb{Z}}\right)_{a}$ (i.e., take congruence $\bmod a$ ) is torsion free, we receive a finite volume hyperbolic manifold and we denote it by $M(\mathcal{Q}, a)$.

We say that $x \in \mathbb{R}^{n+1}$ is an isotropic vector of the quadratic form $\mathcal{Q}$ if $x^{\mathrm{T}} \mathcal{Q} x=0$. Isotropic lines (i.e., lines determined by isotropic vectors) correspond to points in the sphere at infinity of the hyperbolic space $\mathbb{H}^{n}$. (This is easy to see if we use the hyperboloid model of $\mathbb{H}^{n}$.) The following lemma tells us that to a rational isotropic vector of the rational quadratic form $\mathcal{Q}$ of signature $(n, 1)$ corresponds a cusp.

7.1. Lemma. Let $a$ be an integer. If $x \in \mathbb{Q}^{n+1}$ is such that $x^{\mathrm{T}} \mathcal{Q} x=0$, then $\left\{g \in\left(O(\mathcal{Q})_{\mathbb{Z}}\right)_{a}: g x=x, g\right.$ parabolic $\}$ has more than one element. Conversely, if $x$ is isotropic and $\left\{g \in\left(O(\mathcal{Q})_{\mathbb{Z}}\right)_{a}: g x=x, g\right.$ parabolic $\}$ is not the trivial group, then we can choose $x \in \mathbb{Q}^{n+1}$.

Remarks.

7.1.1. The subgroup $\left\{g \in\left(O(\mathcal{Q})_{\mathbb{Z}}\right)_{a}: g x=x, g\right.$ parabolic $\}$ is "the fundamental group of the cusp corresponding to $x "$.

7.1.2. If we delete from $O(\mathcal{Q})_{\mathbb{Z}}$ the elements of finite order (for instance by taking a congruence subgroup), the condition "being parabolic" would be redundant (if $g$ is hyperbolic and fixes a point at infinity corresponding to the isotropic vector $x$, then $x$ is an eigenvector of $g$, but $g x \neq x)$.

Proof of Lemma 7.1. The first part follows from the proof of theorem 5, below (we only use the second part of the lemma in the proof of this theorem). In fact, 
there we give explicit formulas to find a whole subgroup of $\left\{g \in\left(O(\mathcal{Q})_{\mathbb{Z}}\right)_{a}: g x=\right.$ $x, g$ parabolic $\}$ isomorphic to $\mathbb{Z}^{n-1}$.

Suppose then that $\left\{g \in\left(O(\mathcal{Q})_{\mathbb{Z}}\right)_{a}: g x=x, g\right.$ parabolic $\}$ is not the trivial group, and $x^{\mathrm{T}} \mathcal{Q} x=0$. Take $g \in\left\{g \in\left(O(\mathcal{Q})_{\mathbb{Z}}\right)_{a}: g x=x, g\right.$ parabolic $\}, g$ different from the identity $I$. Let $V \subset \mathbb{R}^{n+1}$ be the kernel of $g-I$. Because $g$ is parabolic it acts freely on the hyperboloid $x_{1}^{2}+\ldots+x_{n}^{2}-x_{n+1}^{2}=-1$, which means that $V \cap\left\{y \in \mathbb{R}^{n+1}, y^{\mathrm{T}} \mathcal{Q} y<0\right\}$ is empty. Also, because $x$ is the only isotropic vector fixed by $g$ ( $g$ fixes only one point at infinity) we have that $y^{\mathrm{T}} \mathcal{Q} y>0$ for $y \in V \backslash \mathbb{R} x$. This implies that $\left.\mathcal{Q}\right|_{V}$ is degenerate and $\mathbb{R} x$ is the only line in $V$ such that $y^{\mathrm{T}} \mathcal{Q} x=0$ for all $y \in V$.

On the other hand, because $V$ is the kernel of a matrix with integer coefficients, there is a basis $\left\{z_{1}, \ldots, z_{k}\right\}$ of $V$ where $z_{i} \in \mathbb{Q}^{n+1}$. If $x$ is a multiple of one $z_{i}$, we are done. If not, write $x=\sum \lambda_{i} z_{i}$ and the $\lambda_{i}$ are uniquely determined (modulo multiplication by a constant) by the set of linear equations with rational coefficients: $z_{i}^{\mathrm{T}} \mathcal{Q} x=0, i=1, \ldots, k$. Hence we can assume that the $\lambda_{i}$ 's are rational and this completes the proof of the lemma.

We now prove theorem 5 .

Theorem 5. If $M(\mathcal{Q}, a)$ is as before, then it admits finite covers $M(\mathcal{Q}, b a)$ with one cusp diffeomorphic to $T^{n-1} \times(0, \infty)$.

Proof. Recall that, in general, if $\Sigma$ is a discrete torsion free subgroup of $O(n, 1 ; \mathbb{R})$ such that $\Sigma \backslash O(n, 1 ; \mathbb{R}) / O(n-1, \mathbb{R})$ has finite volume and is non-compact, then the cusps are of the form $F \times[0, \infty)$ with metric $e^{-2 t} S+d t^{2}$, for some flat metric $S$ on the compact manifold $F$ and $F \cong \mathbb{R}^{n-1} / \Lambda$ (see [2] sec. D3), where $\Lambda \subset \Sigma$ is the subgroup of elements that fix one point in the sphere at infinity. Because the points at the sphere at infinity correspond to isotropic lines, we can write $\Lambda=\{g \in \Sigma: g x=x\}$ for some isotropic vector $x$ (see remarks 7.1.1 and 7.1.2).

In our case, because of the lemma above, we can suppose that $M(\mathcal{Q}, a)$ has a cusp that corresponds to $x \in \mathbb{Q}^{n+1}$. If we define $G=\{g \in O(Q): g x=x\}$, to prove the theorem it is enough to prove the following.

7.2. Lemma. $G_{\mathbb{Z}}=G \cap G L(n+1, \mathbb{Z})$ has a congruence subgroup $\left(G_{\mathbb{Z}}\right)_{a}$ isomorphic to $\mathbb{Z}^{n-1}$.

Proof. In what follows, we write $v . w$ instead of $Q(v, w)=v^{\mathrm{T}} Q w$, for $v, w \in \mathbb{R}^{n+1}$. Because $\mathcal{Q}$ is rational of signature $(n, 1)$, there is a basis $\mathcal{B}=\left\{r_{1}, \ldots, r_{n-1}, x_{0}, e\right\} \subset$ $\mathbb{Q}^{n+1}$ for $\mathbb{R}^{n+1}$ satisfying

$$
\begin{aligned}
& r_{1}, \ldots, r_{n-1}, e \in \mathbb{Z}^{n+1}, \\
& x_{0}=\alpha x, \alpha \in \mathbb{Q}, \\
& e . r_{i}=r_{i} . r_{j}=x_{0}^{2}=x_{0} . r_{i}=0 \quad i, j=1, \ldots, n-1 \quad i \neq j, \\
& e^{2}<0, \\
& r_{i}^{2}>0, \\
& x_{0} . e=-1 .
\end{aligned}
$$

Note that if $g \in G$ and $g e=a_{1} r_{1}+\ldots+a_{n-1} r_{n-1}+a_{n} x_{0}+a_{n+1} e$, then $a_{n+1}=1$ because $-1=x_{0} . e=g x_{0} . g e=x_{0} . g e=a_{n+1} x_{0} . e=-a_{n+1}$.

We have four steps.

Step 1. Here we express $G$ as a semidirect product of two subgroups. For each $a=\left(a_{1}, \ldots, a_{n-1}\right) \in \mathbb{R}^{n-1}$, define the linear isomorphism $t_{a}: \mathbb{R}^{n+1} \rightarrow \mathbb{R}^{n+1}$ : 
(a) $t_{a}\left(r_{i}\right)=a_{i} r_{i}^{2} x_{0}+r_{i}, i=1, \ldots, n-1$,

(b) $t_{a}\left(x_{0}\right)=x_{0}$,

(c) $t_{a}(e)=a_{1} r_{1}+\ldots+a_{n-1} r_{n-1}+a_{n} x_{0}+e$, where $a_{n}=\frac{1}{2} \sum_{i=1}^{i=n-1} a_{i}^{2} r_{i}^{2}$.

Then $t_{a}$ written in the basis $\mathcal{B}=\left\{r_{1}, . ., r_{n-1}, x_{0}, e\right\}$ is given by the matrix $\left(t_{a}\right)_{\mathcal{B}}=$ $I+A_{a}$, where $I$ is the identity matrix and

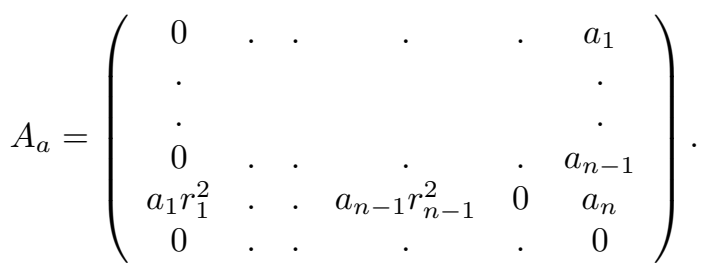

Note that this linear transformation preserves $\mathcal{Q}$ since $t_{a}(\mathcal{B})$ has the same products as $\mathcal{B}$ (for instance, $t_{a}\left(x_{0}\right) \cdot t_{a}(e)=x_{0} \cdot e=-1$ ). Then if $B$ is the matrix whose columns are the canonical coordinates of $r_{1}, \ldots, r_{n-1}, x_{0}, e$ we have that $B\left(t_{a}\right)_{\mathcal{B}} B^{-1} \in G$ and we denote this matrix also by $t_{a}$. It is easy to verify that $t_{a+b}=t_{a} \cdot t_{b}$ so that the map $a \longmapsto t_{a}$ is a homomorphism. Define now

$$
\begin{aligned}
& T=\left\{t_{a}: a \in \mathbb{R}^{n-1}\right\}, \\
& R=\{g \in G: g e=e\} .
\end{aligned}
$$

It is easy to see that $T$ is an abelian subgroup of $G$ isomorphic (as a Lie group) to $\mathbb{R}^{n-1}$ and $T \cap R=\{I\}$.

Note also that $R$ is isomorphic to $O(n-1, \mathbb{R})$ (because $g$ fixes $x_{0}$ and $e$, which implies that $g\left(r_{i}\right)$ is a linear $Q$-orthogonal combination of the $r_{j}$ 's).

Remark that if $g \in G$, and

$$
\left(\begin{array}{c}
a_{0} \\
\cdot \\
\cdot \\
\cdot \\
a_{n-1} \\
a_{n} \\
1
\end{array}\right)=(g e)_{\mathcal{B}}
$$

(i.e., $\left.g e=a_{1} r_{1}+\ldots+a_{n-1} r_{n-1}+a_{n} x_{0}+e\right)$, then $t_{a} e=g e$ where $a=\left(a_{1}, \ldots, a_{n-1}\right)$, so that $t_{a}^{-1} g \in R$. This implies that $G=T R=\{t g: t \in T, g \in R\}$.

Step 2. Here we show that for all but finite primes $p,\left(T_{\mathbb{Z}} R_{\mathbb{Z}}\right)_{p}=\left(T_{\mathbb{Z}}\right)_{p}$.

To see this, note that because $O(Q)_{\mathbb{Z}}$ is discrete, we have that $R_{\mathbb{Z}}$ is also discrete. Then, since $R$ is compact, we get that $R_{\mathbb{Z}}$ is finite, i.e., $R_{\mathbb{Z}}=\left\{I=\rho_{1}, \ldots, \rho_{k}\right\}$, $\rho_{i} \neq I, i \geq 2$. Then every element in $T_{\mathbb{Z}} R_{\mathbb{Z}}$ can be written as $t \rho_{i}$ for some $i=1, \ldots, k$ and $t \in T_{\mathbb{Z}}$.

Now, let $p>2$ be a prime such that

(a) $p$ does not divide $e^{2}$,

(b) $\rho_{i} \not \equiv I(\bmod p), i=2, \ldots, k$,

(c) $p$ does not divide any denominator of the entries of $B$ and $B^{-1}$.

Then we claim that for such a prime $p,\left(T_{\mathbb{Z}} R_{\mathbb{Z}}\right)_{p}=\left(T_{\mathbb{Z}}\right)_{p}$.

To prove this, it is enough to prove $\left(T_{\mathbb{Z}} R_{\mathbb{Z}}\right)_{p} \subset\left(T_{\mathbb{Z}}\right)_{p}$. So, take $t \rho_{i} \in\left(T_{\mathbb{Z}} R_{\mathbb{Z}}\right)_{p}$. Then $t \rho_{i} \equiv I(\bmod p)$. Hence $t e=t \rho_{i} e \equiv e(\bmod p)$, which implies $t e=e+p v$ for some $v \in \mathbb{Z}^{n+1}$. Recall that $t=t_{a}$, where $a=\left(a_{1}, \ldots, a_{n-1}\right)$ is such that 
$t e=a_{1} r_{1}+\ldots+a_{n-1} r_{n-1}+a_{n} x_{0}+e$. Hence $p v=a_{1} r_{1}+\ldots+a_{n-1} r_{n-1}+a_{n} x_{0}$.

But

$$
p v=B B^{-1} p v=B B^{-1}\left(a_{1} r_{1}+\ldots+a_{n-1} r_{n-1}+a_{n} x_{0}\right)=B\left(a_{1}, \ldots, a_{n}, 0\right)
$$

so that $p B^{-1} v=\left(a_{1}, \ldots, a_{n}, 0\right)$.

Consequently, because of (c) above, we have that $a=\left(a_{1}, \ldots, a_{n-1}\right)$ is such that $p$ divides every numerator of the $a_{i}$ 's and does not divide any of its denominators, and the same is true for the entries of the matrix $A_{a}$. Then, because of (c) again, we can see that

$$
t=t_{a}=B\left(t_{a}\right)_{\mathcal{B}} B^{-1}=B\left(I+A_{a}\right) B^{-1}=I+B A_{a} B^{-1} \equiv I(\bmod p) .
$$

Step 3. We show now that we can find congruence subgroups of $G_{\mathbb{Z}}$ contained in $T_{\mathbb{Z}} R_{\mathbb{Z}}$. For this write

$$
\begin{aligned}
B & =\frac{1}{s_{1}} B_{1}, \\
B^{-1} & =\frac{1}{s_{2}} B_{2},
\end{aligned}
$$

where $s_{i} \in \mathbb{Z}$ and $B_{i}$ has integer entries.

Take $m=2 s_{1} s_{2}^{2}$. Let $g \in\left(G_{\mathbb{Z}}\right)_{m}$, that is, $g \equiv I(\bmod m)$. Because of step $1, g=$ $t_{a} \rho_{i}$ and then $t_{a} e=g e$. We show that $t_{a}, t_{a}^{-1} \in T_{\mathbb{Z}}$. Recall that $a=\left(a_{1}, \ldots, a_{n-1}\right)$ is such that $g e=a_{1} r_{1}+\ldots+a_{n-1} r_{n-1}+a_{n} x_{0}+e$. As in step $2, g e=e(\bmod m)$ implies $g e=e+m v$. Then

$$
m v=B B^{-1} m v=B B^{-1}\left(a_{1} r_{1}+\ldots+a_{n-1} r_{n-1}+a_{n} x_{0}\right)=B\left(a_{1}, \ldots, a_{n}, 0\right)
$$

and we have $2 s_{1} s_{2} B_{2} v=m \frac{1}{s_{2}} B_{2} v=m B^{-1} v=\left(a_{0}, \ldots a_{n}, 0\right)$.

Hence $a$ has integer entries. Moreover, we have that $s_{1} s_{2}$ divides all the entries of $a$ so that the same happens with the entries of $A_{a}$ and $A_{-a}$. Then

$$
t_{a}=B\left(t_{a}\right)_{\mathcal{B}} B^{-1}=B\left(I+A_{a}\right) B^{-1}=I+B A_{a} B^{-1}=I+B_{1}\left[\frac{1}{s_{1} s_{2}} A_{a}\right] B_{2} \in T_{\mathbb{Z}}
$$

and analogously for $t_{a}^{-1}=B\left(I+A_{-a}\right) B^{-1}$.

This proves step 3 because

$$
g=t_{a}\left(t_{a}^{-1} g\right) \in T_{\mathbb{Z}} R_{\mathbb{Z}}
$$

Step 4. We complete the proof. Use step 3 to get $\left(G_{\mathbb{Z}}\right)_{m}$ contained in $T_{\mathbb{Z}} R_{\mathbb{Z}}$. Take $p$ coprime to $m$ and apply step 2 to get $\left(G_{\mathbb{Z}}\right)_{m p}=\left(\left(G_{\mathbb{Z}}\right)_{m}\right)_{p} \subset\left(T_{\mathbb{Z}} R_{\mathbb{Z}}\right)_{p}=$ $\left(T_{\mathbb{Z}}\right)_{p}$. (To see that $T_{\mathbb{Z}} \cong \mathbb{Z}^{n-1}$, note that $T_{\mathbb{Z}}$ is a discrete cocompact subgroup of $T \cong \mathbb{R}^{n-1}$.)

7.2.1. Remark. Note that step 4 above implies $\left(G_{\mathbb{Z}}\right)_{m p} \subset\left(T_{\mathbb{Z}}\right)_{m p}$. From this and $T \subset G$, it follows that $\left(G_{\mathbb{Z}}\right)_{m p}=\left(T_{\mathbb{Z}}\right)_{m p}$, for all but finite primes $p$. Also note that if $a \in \mathbb{Z}$ is such that $\left(G_{\mathbb{Z}}\right)_{a}=\left(T_{\mathbb{Z}}\right)_{a}$, then, for every $c \in \mathbb{Z}$, we have $\left(G_{\mathbb{Z}}\right)_{a c}=\left(T_{\mathbb{Z}}\right)_{a c}$. (This is because $\left(G_{\mathbb{Z}}\right)_{a}=\left(T_{\mathbb{Z}}\right)_{a}$ implies $\left(G_{\mathbb{Z}}\right)_{a c}=\left(\left(G_{\mathbb{Z}}\right)_{a}\right)_{a c}=\left(\left(T_{\mathbb{Z}}\right)_{a}\right)_{a c}=\left(T_{\mathbb{Z}}\right)_{a c} \cdot$ )

7.3. Corollary. $M(\mathcal{Q}, a)$ has a finite cover $M(\mathcal{Q}, b a)$, with all cusps diffeomorphic to $T^{n-1} \times(0, \infty)$.

Proof. Recall that to an isotropic rational vector $x$ corresponds a cusp. But different rational isotropic vectors may correspond to the same cusp. In fact, if $x$ determines a cusp, then the set of all isotropic rational vectors determining the same cusp is $\{g x$ : $g \in \Gamma\}$, where $\Gamma=\left(O(\mathcal{Q})_{\mathbb{Z}}\right)_{a}$ is the fundamental group of the manifold $M(\mathcal{Q}, a)$. 
The problem is that after taking finite covers, $x$ and $g x$ may not correspond to the same cusp anymore. Given $y \in \mathbb{R}^{n+1}$, let $G^{y}=\{g \in O(\mathcal{Q}): g y=y\}$. Because we have a finite number of cusps, what we have to prove is that if $a$ is such that $\left(\left(G^{x}\right)_{\mathbb{Z}}\right)_{a} \cong \mathbb{Z}^{n-1}$, then $\left(\left(G^{g x}\right)_{\mathbb{Z}}\right)_{a} \cong \mathbb{Z}^{n-1}$, for $g \in \mathcal{O}(Q)_{\mathbb{Z}}$. For this just note that $g^{-1} G^{g x} g=G^{x}$. Thus, if $g \in \mathcal{O}(Q)_{\mathbb{Z}}$, then $g^{-1}\left(\left(G^{g x}\right)_{\mathbb{Z}}\right)_{a} g \subset\left(\left(G^{x}\right)_{\mathbb{Z}}\right)_{a} \cong \mathbb{Z}^{n-1}$. The corollary follows.

7.4. Corollary. Suppose that $M(\mathcal{Q}, a)$ has a cusp diffeomorphic to $T^{n-1} \times[0, \infty)$. Then there are integers $b$ and $q_{0}$ such that if $q \geq q_{0},(q, b a)=1$, we have that $M(\mathcal{Q}, q b a)$ has a large cusp.

Proof. We use all notation from the proof of theorem 5. Recall that we have a map $t: \mathbb{R}^{n-1} \rightarrow G, a \mapsto t_{a}$.

Claim. If $c$ is such that $t^{-1}\left(\left(T_{\mathbb{Z}}\right)_{c}\right) \subset \mathbb{Z}^{n-1}$, then $t^{-1}\left[\left(T_{\mathbb{Z}}\right)_{c q}\right]=t^{-1}\left[\left(T_{\mathbb{Z}}\right)_{c}\right] \cap[q \mathbb{Z}]^{n-1}$, where $q$ is large enough and coprime to $c$.

Proof of the claim. If $a \in t^{-1}\left[\left(T_{\mathbb{Z}}\right)_{c q}\right]$, then $t_{a} \in\left(T_{\mathbb{Z}}\right)_{c q}$ and we have $t_{a} \in\left(T_{\mathbb{Z}}\right)_{c}$. We have to prove that $a \in[q \mathbb{Z}]^{n-1}$. For this, note that we also have $t_{a} \in\left(T_{\mathbb{Z}}\right)_{q}$, which implies $B A_{a} B^{-1} \equiv 0(\bmod q)$, and, by taking $q$ large enough (so that $q$ does not divide any denominator of the entries of $B$ and $B^{-1}$ ), we have that $q$ divides every entry of $A_{a}$, so that $q$ divides every entry of $a$.

Conversely, assume $a \in t^{-1}\left[\left(T_{\mathbb{Z}}\right)_{c}\right] \cap[q \mathbb{Z}]^{n-1}$. Then $t_{a} \in\left(T_{\mathbb{Z}}\right)_{c}$. On the other hand, because $a \in[q \mathbb{Z}]^{n-1}, A_{a} \equiv 0(\bmod q)$ and proceeding as before, we get $t_{a} \equiv I(\bmod q)$. But $(q, c)=1$, and hence from $t_{a} \equiv I(\bmod c)$ and $t_{a} \equiv I(\bmod q)$, we get $t_{a} \equiv I(\bmod c q)$. This proves the claim.

Recall from remark 7.2.1 that there is a $b$ such that $\left(G_{\mathbb{Z}}\right)_{b a}=\left(T_{\mathbb{Z}}\right)_{b a}$. Also, it is not difficult to see that we can take $b$ such that $t^{-1}\left(\left(T_{\mathbb{Z}}\right)_{b}\right) \subset \mathbb{Z}^{n-1}$. These observations together with the claim imply (take $c=b a$ in the claim) $t^{-1}\left[\left(G_{\mathbb{Z}}\right)_{(b a) q}\right]=$ $t^{-1}\left[\left(G_{\mathbb{Z}}\right)_{(b a)}\right] \cap[q \mathbb{Z}]^{n-1}$, where $(b a, q)=1$.

Now, if $\left\{v_{1}, \ldots, v_{n-1}\right\}$ is a basis for $t^{-1}\left[\left(G_{\mathbb{Z}}\right)_{(b a)}\right]$, we claim that $\left\{q v_{1}, \ldots, q v_{n-1}\right\}$ is a basis for $t^{-1}\left[\left(G_{\mathbb{Z}}\right)_{(b a) q}\right]$. For this take $q>\operatorname{det} E$, where $E$ is the matrix whose columns are the (integer) coordinates of the $v_{i}$ 's. Then $\operatorname{det} E \not \equiv 0(\bmod q)$. Now if $x \in t^{-1}\left[\left(G_{\mathbb{Z}}\right)_{(b a)}\right] \cap[q \mathbb{Z}]^{n-1}$, then $x=\sum \lambda_{i} v_{i}$ and we have to prove that $q$ divides all $\lambda_{i}$ 's. But $x \in[q \mathbb{Z}]^{n-1}$, and hence $E \lambda \equiv 0(\bmod q)$, where $\lambda=\left(\lambda_{1}, \ldots, \lambda_{n-1}\right)$, and because $\operatorname{det} E \not \equiv 0(\bmod q) E$ has an inverse in $\mathbb{Z}_{q}(q$ is prime) and we get $\lambda \equiv 0(\bmod q)$.

This means that the coverings obtained are simple (with respect to $\left\{v_{1}, \ldots, v_{n-1}\right\}$ ) and, because we can take $q$ as large as we want, the corollary follows from lemma 2.14 .

Let $T^{1}=\left\{t_{s e_{1}}: s \in \mathbb{R}\right\}$, that is, $T_{1}$ is the subgroup of $T$ generated by $t_{e_{1}}$. Also, let $T^{2}=\left\{t_{y}: y \in\{0\} \times \mathbb{R}^{n-2}\right\}$.

7.5. Lemma. If the isotropic vector $x_{0}$ has a zero first coordinate (i.e., $x_{0}=$ $(0, *, *, \ldots, *))$ and $\mathcal{Q}$ is diagonal of the form $\lambda_{1} x_{1}^{2}+\ldots+\lambda_{n} x_{n}^{2}-\lambda_{n+1} x_{n+1}^{2}, \lambda_{i}>0$, then, for any integer $k \neq 0$, we have

$$
\left(T_{\mathbb{Z}}\right)_{2 k}=\left(T_{\mathbb{Z}}^{1}\right)_{2 k} \oplus\left(T_{\mathbb{Z}}^{2}\right)_{2 k} .
$$

Proof. We will use the notation of the proof of lemma 7.2. Because the first coordinate of $x_{0}$ is zero, we can take $r_{1}=e_{1}=(1,0, \ldots, 0)$, and $r_{i}, r \geq 2$ and $e$ having 
their first coordinates equal to zero. Then the matrix $B$, whose columns are the $r_{1}, \ldots, r_{n-1}, x_{0}, e$ can be written as $\left(\begin{array}{cc}1 & 0 \\ 0 & B_{0}\end{array}\right)$, where the columns of $B_{0}$ are the $\left\{r_{2}, \ldots, r_{n-1}, x_{0}, e\right\}$, with the first coordinates (which are zero) deleted.

Let $a \in \mathbb{R}^{n-1}$. Then $a=a_{1} e_{1}+b, a_{1} \in \mathbb{R}, b \in\{0\} \times R^{n-2}$. Thus $t_{a}=t_{a_{1} e_{1}} t_{b}$ (recall that $a \mapsto t_{a}$ is a homomorphism). Now, for any integer $l$, we have $t_{a} \equiv$ $I(\bmod l)$ iff $I+B A_{a} B^{-1} \equiv I(\bmod l)$ iff $B A_{a} B^{-1} \equiv 0(\bmod l)$. But $B=\left(\begin{array}{cc}1 & 0 \\ 0 & B_{0}\end{array}\right)$ and $A_{a}=A_{a_{1} e_{1}}+A_{b}$, and a straightforward calculation shows

$$
B A_{a} B^{-1}=\left(\begin{array}{cc}
0 & a_{1} y \\
a_{1} e_{1}^{2} x_{0} & B_{0} A_{b} B_{0}^{-1}+C
\end{array}\right)
$$

where $y$ is the last line of $B_{0}^{-1}$ and $C=\left(\frac{1}{2} a_{1}^{2} e_{1}^{2}\right) x_{0} y$ (note that $x_{0}$ is an $n \times 1$ matrix and $y$ is a $1 \times n$ matrix).

Hence, if $B A_{a} B^{-1} \equiv 0(\bmod l)$, then $a_{1} e_{1}^{2} x_{0} \equiv 0(\bmod l)$ and $a_{1} y \equiv 0(\bmod l)$. This implies, together with $C=\left(\frac{1}{2} a_{1}^{2} e_{1}^{2}\right) x_{0} y$ and $l=2 k$, that $C \equiv 0(\bmod 2 k)$ and $B_{0} A_{b} B_{0}^{-1} \equiv 0(\bmod 2 k)$. Conversely, if $B_{0} A_{b} B_{0}^{-1} \equiv 0(\bmod 2 k), a_{1} e_{1}^{2} x_{0} \equiv$ $0(\bmod 2 k)$ and $a_{1} y \equiv 0(\bmod 2 k)$, we get $B A_{a} B^{-1} \equiv 0(\bmod 2 k)$. Consequently, we have the equivalence $B A_{a} B^{-1} \equiv 0(\bmod 2 k)$ iff $B A_{a_{1} e_{1}} B^{-1} \equiv 0(\bmod 2 k)$ and $B A_{b} B^{-1} \equiv 0(\bmod 2 k)$. Equivalently, we get $t_{a} \equiv I(\bmod 2 k)$ iff $t_{a_{1} e_{1}} \equiv I(\bmod 2 k)$ and $t_{b} \equiv I(\bmod 2 k)$. This proves the lemma.

Let $J=\operatorname{diag}\{-1,1,1, \ldots, 1\}$. The proof of the following lemma is a straightforward calculation.

7.6. Lemma. Consider the involution $\iota: T_{\mathbb{Z}} \rightarrow T_{\mathbb{Z}}$, given by $\iota(g)=J g J$. Then, under the same assumptions as lemma 7.5, we have

(i) $f i x(\iota)=T_{\mathbb{Z}}^{2}$,

(ii) $\iota\left(t_{e_{1}}\right)=t_{-e_{1}}=t_{e_{1}}^{-1}$.

Note that $g \equiv I(\bmod a)$ iff $\iota(g) \equiv I(\bmod a)$. Hence, we can consider $\iota:\left(G_{\mathbb{Z}}\right)_{a} \rightarrow$ $\left(G_{\mathbb{Z}}\right)_{a}$, for any $a \in \mathbb{Z}$. The next corollary follows from lemmas 7.5 and 7.6 , remark 7.2.1 and 4.6.1.

7.8. Corollary. Under the same assumptions as lemma 7.5, we have that there is $a \in \mathbb{Z}$, such that for all $c \in \mathbb{Z}, \iota:\left(G_{\mathbb{Z}}\right)_{a c} \rightarrow\left(G_{\mathbb{Z}}\right)_{a c}$ is simple.

\section{Proof of Theorem 6}

Theorem 6. Let $M(\mathcal{Q}, a)$ be as before with $\mathcal{Q}$ satisfying 0.1 . Then $M(\mathcal{Q}, a)$ admits finite covers $M_{0}$, such that there are non-compact finite volume real hyperbolic manifolds $M_{1}, M_{2}$ satisfying the following conditions:

(1) $\operatorname{dim} M_{0} \geq 5, \operatorname{dim} M_{1}=n-1, \operatorname{dim} M_{2}=n-3$;

(2) $M_{2} \subset M_{1} \subset M_{0}$ and the inclusions are totally geodesic;

(3) the normal bundle of $M_{2}$ in $M_{0}$ is trivial;

(4) $M_{0}$ has a large cusp $C_{M_{0}}$ diffeomorphic to $T^{n-1} \times(0, \infty)$;

(5) $M_{i}$ has exactly one cusp contained in $C_{M_{0}}, i=1,2$.

Proof. Let $\mathcal{Q}$ be a diagonal rational quadratic form of signature $(n, 1)$,

$$
Q\left(x_{1}, \ldots, x_{n+1}\right)=\lambda_{1} x_{1}^{2}+\ldots+\lambda_{n} x_{n}^{2}-\lambda_{n+1} x_{n+1}^{2}, \quad \lambda_{i} \in \mathbb{Q}
$$

with $\lambda_{i}>0$, and $\lambda_{1}=\lambda_{2}=\lambda_{3}$. 
By (0.1), if $n \leq 6, \lambda_{4} x_{4}^{2}+\ldots+\lambda_{n} x_{n}^{2}-\lambda_{n+1} x_{n+1}^{2}$ has a rational isotropic vector. Denote this vector by $\left(0,0,0, z_{4}, \ldots, z_{n}, z_{n+1}\right)$.

If $n \geq 7$, the form

$$
\lambda_{4} x_{4}^{2}+\ldots+\lambda_{n-2} x_{n-2}^{2}+\lambda_{n-1} x_{n-1}^{2}+\lambda_{n} x_{n}^{2}-\lambda_{n+1} x_{n+1}^{2}
$$

has, by the Hasse-Minkowski Theorem, a rational isotropic vector. Denote this vector also by $\left(0,0,0, z_{4}, \ldots, z_{n}, z_{n+1}\right)$. In any case, our rational isotropic vector has, at least, the first three coordinates equal to zero.

Now, $M(\mathcal{Q}, a)$ (for all but a finite number of primes $a$ ) has a cusp $C_{M}$ corresponding to that isotropic vector (see lemma 7.2).

Because of theorem 5 we can assume that $C$ is diffeomorphic to $T^{n-1} \times(0, \infty)$.

Now, by [14], $M(\mathcal{Q}, a)$ has a finite orientable cover $M=M(\mathcal{Q}, b a)$ with three totally geodesic orientable hypersurfaces $H_{i}, \quad i=1,2,3$, that do not separate $M$ and are contained in the fixed point set of an isometric involution $\iota_{i}$. In fact, if $p_{M}: \mathbb{I}^{n} \rightarrow$ $M$ is the covering projection $\left(\mathbb{I}^{n} \subset \mathbb{R}^{n+1}\right.$ is the hyperboloid model of the hyperbolic space $\left.\mathbb{H}^{n}\right)$, then $H_{i}=p_{M}\left(\left\{x_{i}=0\right\}\right)$, where $\left\{x_{i}=0\right\}=\mathbb{I}^{n} \cap\left\{\left(x_{1}, \ldots, x_{n+1}\right) \in \mathbb{R}^{n+1}\right.$ : $\left.x_{i}=0\right\}$. (To obtain $H_{i}, i=1,2,3$, apply [14] to each involution $\iota_{i}: \mathbb{R}^{n+1} \rightarrow \mathbb{R}^{n+1}$, $\iota_{i}\left(x_{1}, \ldots, x_{i}, \ldots, x_{n+1}\right)=\left(x_{1}, \ldots,-x_{i}, \ldots, x_{n+1}\right)$ fixing $\left\{x_{i}=0\right\}$. For each $i=1,2,3$, [14] gives a congruence subgroup. Take the intersection.) Note that the $H_{i}$ 's are mutually orthogonal.

By [14] again we can assume also that $N=p_{M}\left(\left\{x_{1}=x_{2}=x_{3}=0\right\}\right) \subset H_{1} \cap H_{2} \cap$ $H_{3}$ is a totally geodesic submanifold of $M$. (Here note that $\iota\left(x_{1}, x_{2}, x_{3}, x_{4}, \ldots, x_{n+1}\right.$ ) $=\left(-x_{1},-x_{2},-x_{3}, x_{4}, \ldots, x_{n+1}\right)$ fixes $\left\{x_{1}=x_{2}=x_{3}=0\right\}$ and use lemma 2.1 of [14.) We have $N \subset H_{i} \subset M, i=1,2,3$, and all the inclusions are totally geodesic.

Then the $H_{i}$ 's and $N$ are non-compact and have a cusp corresponding to the same rational isotropic vector $\left(0,0,0, z_{4}, \ldots, z_{n-2}, z_{n-1}, z_{n}, z_{n+1}\right)$. Hence $H_{i}, i=1,2,3$, all have at least one cusp $C_{H_{i}}$ contained in $C_{M}$. By corollary 7.4, we can assume $C_{M}$ to be large. (Note that the properties of $p_{M}\left(\left\{x_{i}=0\right\}\right)$ being a manifold, being contained in the fixed point set of an isometric involution, not separating $M$, and having the cusp corresponding to $\left(0,0,0, z_{4}, \ldots, z_{n}, z_{n+1}\right)$ with torus cross section, are not destroyed by taking further congruence subgroups.)

8.1. Note that, because $\lambda_{i}>0$ and $\lambda_{1}=\lambda_{2}=\lambda_{3}$, we have that, for $i, j \in\{1,2,3\}$, $i \neq j$, there is an isometry $f: M \rightarrow M$, with $f H_{i}=H_{j}$. (For example, for $i=1$, $j=2$, we can take $f$ induced by $\left(x_{1}, x_{2}, x_{3}, \ldots, x_{n+1}\right) \mapsto\left(x_{2}, x_{1}, x_{3}, \ldots, x_{n+1}\right)$.)

Let $T_{M}$ denote the flat torus cross section of $C_{M}$, and consider the restriction $\left.\bar{\iota}_{i}\right|_{T_{M}}: T_{M} \rightarrow T_{M}$, of the isometric involution $\overline{\iota_{i}}: M \rightarrow M$, induced by $\iota_{i}:$ $\mathbb{R}^{n+1} \rightarrow \mathbb{R}^{n+1}$, defined above. (Note that $H_{i}$ is contained in the fixed point set of an involution, and this involution sends $C_{M}$ to itself; hence, the involution induces an isometric involution on the cross section $T_{M}$ of the cusp $C_{M}$.) Then corollary 7.8 implies that we can suppose $\bar{\iota}_{i}$ is simple (note that, for instance, $\bar{\iota}_{1}: M \rightarrow M$ at the level of matrices is given by conjugation with the matrix $J=\operatorname{diag}\{-1,1, \ldots, 1\}$ ).

We have two cases.

First case. Suppose $H_{1}$ has exactly one cusp in $C_{M}$.

In this case, by 8.1, the same happens with $H_{2}$ and $H_{3}$. Let $T_{H_{i}}$ be the corresponding tori cross sections of the (only) cusps of $H_{i}$ contained in $C_{M}$. Again by 8.1, and the fact that the isometries induced by the $\iota_{i}$ are simple, we get that each $T_{H_{i}}$ is simple in $T_{M}$. Hence, from the definition of simple subtorus and lemma 4.7, 
we get that $T_{M} \cap N \subset \bigcap_{i=1}^{3} T_{H_{i}}$ is connected. Hence, $N$ has exactly one cusp in $C_{M}$. This proves the theorem in this case.

Second case. Suppose $H_{1}$ has more than one cusp in $C_{M}$.

Recall that $H_{i}$ is contained in the fixed point set of an involution. Hence, by lemma 4.6, $H_{1}$ has exactly two cusps in $C_{M}$.

Let $\alpha_{i}$ be the element in $\pi_{1}(M)$ representing the orthogonal $\mathbb{S}^{1}$ to $T_{H_{i}}$ (see 4.6). Because $\pi_{1}(M)$ is residually finite, there is a homomorphism from $\pi_{1}(M)$ to a finite group such that the image of each $\alpha_{i}$ has order larger than two, hence is not zero (in our case this is simple, for we can take a prime $p$ not dividing one of the nondiagonal entries of the integer matrix corresponding to $\alpha_{i}$ and take congruence $\bmod p$ ). Take the finite cover corresponding to the kernel of this homomorphism and call this cover $M^{\prime}$ and choose a cusp $C_{M^{\prime}}$ that maps to $C_{M}$ by the covering projection (note that we can choose $p$ to be very large so that $C_{M^{\prime}}$ is still large; see corollary 7.4). Now, the pre-image of $C_{H_{i}}$ by the covering projection intersected with $C_{M^{\prime}}$ gives many cusps (more than one because we are unwrapping all $\alpha_{i}$ 's). Let $H_{i}^{\prime}=p_{M^{\prime}}\left(\left\{x_{i}=0\right\}\right)$, so that $H_{i}^{\prime}$ maps to $H_{i}$ by the covering projection. If one $H_{i}^{\prime}$ has exactly one cusp in $C_{M^{\prime}}$, by the first case, we are done. So, we assume that $H_{1}$ (hence $H_{i}, i=1,2,3$ ) has more than one cusp in $C_{M^{\prime}}$. Recall that we can still suppose that $H_{i}^{\prime}$ is in the fixed point set of an isometric involution of $M^{\prime}$, so that $H_{i}^{\prime}$ also has exactly two cusps in $C_{M^{\prime}}$. Note that the pre-image of $C_{H_{i}}$ by the covering projection intersected with $C_{M^{\prime}}$ has more than one component so that there is another connected $H_{i}^{\prime \prime}$, whose image is also $H_{i}$ by the covering projection, that has a cusp in $C_{M^{\prime}}$ (that maps to $C_{H_{i}}$ ). But the covering is normal (congruence subgroups are normal subgroups) so that the covering transformations are transitive in the fibers, which implies that there is a covering transformation sending $H_{i}^{\prime}$ to $H_{i}^{\prime \prime}$. Hence $H_{i}^{\prime \prime}$ has also two cusps in $C_{M^{\prime}}$. Note that $H_{i}^{\prime}$ and $H_{i}^{\prime \prime}$ do not intersect.

Now, we construct, for each $i=1,2,3$, the double cover corresponding to $H_{i}^{\prime \prime}$. Since $H_{i}^{\prime}$ (and then $H_{i}^{\prime \prime}$ ) does not separate $M^{\prime}$, there is a closed path $\beta_{i}$ that intersects $H_{i}^{\prime \prime}$ in one point, which means that it is not zero in $H_{1}\left(M^{\prime}, \mathbb{Z}_{2}\right)$ so that the composite

$$
\pi_{1}\left(M^{\prime}\right) \rightarrow H_{1}\left(M^{\prime}, \mathbb{Z}_{2}\right) \rightarrow \mathbb{Z}_{2}
$$

(the last map sends $\beta_{i}$ to one) gives a non-zero homomorphism whose kernel has index two, which corresponds to a double cover. We can also do this geometrically. Given $i=1,2,3$, take two copies of $M^{\prime}, M_{i}^{\prime}(1), M_{i}^{\prime}(2)$. Cut along $H_{i}^{\prime \prime}(j)$ in $M_{i}^{\prime}(j)$, $j=1,2$, so that we end up with four copies of $H_{i}^{\prime \prime}: H_{i}^{\prime \prime}(1)^{+}$and $H_{i}^{\prime \prime}(1)^{-}$from $M_{i}^{\prime}(1)$ and $H_{i}^{\prime \prime}(2)^{+}$and $H_{i}^{\prime \prime}(2)^{-}$from $M_{i}^{\prime}(2)$. Identify $H_{i}^{\prime \prime}(1)^{+}$with $H_{i}^{\prime \prime}(2)^{-}$and $H_{i}^{\prime \prime}(1)^{-}$with $H_{i}^{\prime \prime}(2)^{+}$. These are the double covers $M_{i}^{\prime \prime}$ of $M^{\prime}$ corresponding to $H_{i}^{\prime \prime}$ (note that it is connected because $H_{i}^{\prime \prime}$ does not separate $M^{\prime}$ ) and since $H_{i}^{\prime}$ does not intersect $H_{i}^{\prime \prime}$, the pre-image of $H_{i}^{\prime}$ by this double cover is just two disjoint copies of $H_{i}^{\prime}$. Note also that if we take one point in each cusp of $H_{i}^{\prime}$ inside $C_{M^{\prime}}$ we can join these two points by one path in $H_{i}^{\prime}$ (so that it does not intersect $H_{i}^{\prime \prime}$ ) and we can join them also by another path inside $C_{M^{\prime}}$ that intersects $H_{i}^{\prime \prime}$ once. By joining these two paths, we obtain a loop that intersects $H_{i}^{\prime \prime}$ once so that its lifting begins in one cusp and ends in another. This proves that any pre-image of $H_{i}^{\prime}$ has just one cusp in a cusp of the double cover constructed. Note that, because we are not unwrapping any loop in the cusp $C_{M^{\prime}}$, we still have that every pre-image of $C_{M^{\prime}}$ is still large. (In fact, we have two pre-images of $C_{M^{\prime}}$ each isometric to $C_{M^{\prime}}$, which in turn implies $\pi_{1}\left(C_{M^{\prime}}\right) \subset \pi_{1}\left(M_{i}^{\prime \prime}\right)$.) 
Let $\Gamma=\left(\pi_{1} M_{1}^{\prime \prime}\right) \cap\left(\pi_{1} M_{2}^{\prime \prime}\right) \cap\left(\pi_{1} M_{3}^{\prime \prime}\right) \subset \pi_{1} M^{\prime}$. Then, $\Gamma$ is of finite index in $\pi_{1} M^{\prime}$, and $\pi_{1} C_{M^{\prime}} \subset \Gamma$ (because $\left.\pi_{1} C_{M^{\prime}} \subset \pi_{1} M_{i}^{\prime \prime}, i=1,2,3\right)$. Let $M_{0}=\mathbb{H}^{n} / \Gamma$, $M_{1}^{i}=p_{M_{0}}\left(\left\{x_{i}=0\right\}\right), i=1,2,3$ and $M_{2}=p_{M_{0}}\left(\left\{x_{1}=x_{2}=x_{3}=0\right\}\right)$. We have that $M_{1}=M_{1}^{1}$ has exactly one cusp in $C_{M_{0}}$, with cross section $T_{M_{i}^{1}}$; and, because the projection map $C_{M_{0}} \rightarrow C_{M^{\prime}}$ is an isometry, each $T_{M_{i}^{1}}$ is simple in $C_{M_{0}}$ (recall that each $T_{M_{i}^{\prime}}$ is simple in $T_{M^{\prime}}$ ). Then, lemma 4.7 implies that $M_{2}$ has exactly one cusp in $M_{0}$. This completes the proof.

\section{Proof of Theorem 2}

Here we prove theorem 2, but first we need some notation and remarks. Let $M$ be a non-compact finite volume real hyperbolic manifold, with cusps $F_{i} \times[0, \infty), i=$ $1, \ldots, k$, where the $F_{i}$ 's are flat manifolds. Consider, for each $i$, the totally geodesic embedding $F_{i} \subset D M$, where the curvature outside $\bigcup F_{i}$ is strictly negative (see section 1).

Note now that $\mathbb{R}^{n}$ is (diffeomorphic to) the universal cover of $D M$, and call the covering projection $p$. Then $p^{-1}\left(\bigcup F_{i}\right)$ is a disjoint union of closed hypersurfaces of $\mathbb{R}^{n}$, each diffeomorphic to $\mathbb{R}^{n-1}$; hence each divides $\mathbb{R}^{n}$ into two components having the hypersurface as common boundary. Denote by $\mathcal{W}$ the set of all these hypersurfaces (then $\bigcup \mathcal{W}=p^{-1}\left(\bigcup F_{i}\right)$ ) and call each element of $\mathcal{W}$ a wall.

Recall that we have two canonical embeddings of $M^{0}=M \backslash \bigcup\left\{F_{i} \times(b, \infty)\right\}$, for some $b$, in $D M, j_{i}: M^{0} \rightarrow D M, i=1,2$, and $j_{1}\left(M^{0}\right) \cap j_{2}\left(M^{0}\right)=j_{1}\left(\partial M^{0}\right)=$ $j_{2}\left(\partial M^{0}\right)=\bigcup F_{i}$. Then $p^{-1}\left(j_{i}\left(\right.\right.$ int $\left.\left.M^{0}\right)\right), i=1,2$, is a disjoint union of open subsets of $\mathbb{R}^{n}$, all diffeomorphic to $\mathbb{R}^{n}$, with boundary a union of walls. Call them regions. Note that each wall is in the boundary of exactly two regions, one in $p^{-1}\left(j_{1}\left(M^{0}\right)\right)$ and the other in $p^{-1}\left(j_{2}\left(M^{0}\right)\right)$.

Choose now $x_{i} \in F_{i} \subset D M$ and $\tilde{x}_{i} \in \mathbb{R}^{n}$ such that $p\left(\tilde{x}_{i}\right)=x_{i}$, and this gives a correspondence (one for each $i$ ) between $\pi_{1}\left(D M, x_{i}\right)$ and the deck transformations on $\mathbb{R}^{n}$. Under this correspondence we have the following:

$$
g \in \pi_{1}\left(F_{i}, x_{i}\right) \subset \pi_{1}\left(D M, x_{i}\right) \text { if and only if } g W_{i}=W_{i}, \quad \text { for } i=1, \ldots, k .
$$

where $W_{i}$ is the wall mapping to $F_{i}$ and containing $\tilde{x}_{i}$. We now have a lemma.

9.2. Lemma. Let $g$ be a deck transformation such that $\left(g^{\ell}\right) W=W$ for some wall $W$ and some integer $\ell$. Then $g W=W$.

Proof. First we have a claim.

Claim. Let $z: \mathbb{R} \rightarrow \mathbb{R}^{n}$ be a geodesic $\left(\mathbb{R}^{n}\right.$ with the metric pulled back from $D M$ ) contained in a wall $W$. Then $d\left(z(t), W_{0}\right) \rightarrow \infty$, as $t \rightarrow \infty$, for $W_{0} \neq W$.

Proof of the claim. Choose a point $x \in W_{0}$. Then if $z^{\prime}$ is a geodesic passing through $x$ and contained in $W_{0}$, [13] implies that inf $\left\{d\left(z(t), z^{\prime}(r)\right) ; r \in \mathbb{R}\right\} \rightarrow \infty$, as $t \rightarrow \infty$ (there is no flat curvature outside the walls). But the set of all geodesic rays in $W_{0}$ beginning at $x$ is parametrized by the sphere $S^{n-1}$, which is compact, so that the claim follows because every point in $W_{0}$ can be joined to $x$ by a geodesic.

Define now $f: \mathbb{R}^{n} \rightarrow \mathbb{R}$ by $f(p)=\sum_{j=1}^{\ell} d\left(p,\left(g^{j}\right) W\right)$. Then $f$ is continuous and because the distance between walls goes to infinity as we approach infinity (this follows from the claim above) $f$ has a minimum value at a point $p_{0}$. Now, if $g W \neq$ $W$, then $p_{0}$ is unique (unique because $f$ is now strictly convex, i.e., $\frac{d^{2} f \gamma(t)}{d t^{2}}>0$, for 
all geodesics $\gamma)$. But $f\left(g p_{0}\right)=\sum_{j=1}^{\ell} d\left(g p_{0},\left(g^{j}\right) W\right)=\sum_{j=1}^{\ell} d\left(p_{0},\left(g^{j}\right) W\right)=f\left(p_{0}\right)$, and hence $p_{0}=g p_{0}$, a contradiction because $g$ acts freely. This completes the proof of lemma 9.2 .

Because the $F_{i}$ are flat manifolds, each $F_{i}$ has a flat torus finite cover $q_{i}: T_{i}^{n-1} \rightarrow$ $F_{i}$. Denote the image of $\pi_{1}\left(T_{i}^{n-1}\right) \cong \mathbb{Z}^{n-1}$ in $\pi_{1}\left(D M, x_{i}\right)$ by the map $\pi_{1}\left(T_{i}^{n-1}\right) \stackrel{\left(q_{i}\right)_{*}}{\rightarrow}$ $\pi_{1}\left(F_{i}, x_{i}\right) \rightarrow \pi_{1}\left(D M, x_{i}\right)$ by $Z_{i}$. Recall that there is curvature zero only at the $F_{i}$ 's; hence, because of the torus theorem of 13 and because $n>2$, the only subgroups of $\pi_{1}(D M, *)$ isomorphic to $\mathbb{Z}^{n-1}$ are the conjugates of the $\beta^{-1} Z_{i} \beta$ 's, where $\beta$ is a path joining base points. Note that, for $i \neq j, Z_{i}$ and $Z_{j}$ do not induce conjugate subgroups. (Proof: if they were conjugate, the immersions $T^{n-1} \rightarrow F_{i} \rightarrow D M$ and $T^{n-1} \rightarrow F_{j} \rightarrow D M$ would be homotopic (because $F_{i}, F_{j}$ and $D M$ are aspherical) and their lifts would be at bounded distance. Hence, by the claim above, they would be equal.)

We now prove theorem 2.

Theorem 2. Let $f: D M \rightarrow D M$ be a homeomorphism, where $M$ is a non-compact finite volume real hyperbolic $n$-manifold, $n>2$. Then $f \sim g$, where $g: D M \rightarrow D M$ is a diffeomorphism.

Proof. Let $f: D M \rightarrow D M$ be a homeomorphism and let $\tilde{f}: \mathbb{R}^{n} \rightarrow \mathbb{R}^{n}$ be a lifting, that is, $f p=p \tilde{f}$. We have that $f_{*}: \pi_{1}(D M, *) \rightarrow \pi_{1}(D M, f(*))$ permutes the conjugacy classes corresponding to the $Z_{i}$ 's. Hence we get a bijection $f$ : $\{1, \ldots, k\} \rightarrow\{1, \ldots, k\}$ such that $f_{*} Z_{i}=\beta^{-1} Z_{f(i)} \beta$, where $\beta$ is a certain path joining $f\left(x_{i}\right)$ and $x_{f(i)}$. By moving $f$ by an isotopy, we can suppose then that $f\left(x_{i}\right)=x_{f(i)}$. Moreover, we can suppose that $f_{*} Z_{i}=Z_{f(i)}$, for we could obtain any conjugation by moving the base point $x_{f(i)}$ around a closed path, again by an isotopy.

9.3. Claim. $f_{*}\left(\pi_{1}\left(F_{i}, x_{i}\right)\right)=\pi_{1}\left(F_{f(i)}, x_{f(i)}\right)$.

To see this, take $g \in \pi_{1}\left(F_{i}, x_{i}\right)$. Because $Z_{i}$ has finite index in $\pi_{1}\left(F_{i}, x_{i}\right)$, we have that $g^{\ell} \in Z_{i}$ for some integer $\ell$. Then $f_{*}\left(g^{\ell}\right) \in Z_{f(i)} \subset \pi_{1}\left(F_{f(i)}, x_{f(i)}\right)$, and, by remark $9.1, f_{*}\left(g^{\ell}\right) W_{f(i)}=W_{f(i)}$. But $f_{*}\left(g^{\ell}\right)=\left(f_{*} g\right)^{\ell}$ and lemma 9.2 implies $\left(f_{*} g\right) W_{f(i)}=W_{f(i)}$. Hence, by 9.1 again, $f_{*} g \in \pi_{1}\left(F_{f(i)}, x_{f(i)}\right)$. This proves one inclusion and, proceeding in the same way with $f^{-1}$, we get the other inclusion. This proves claim 9.3. Note that this result proves, in particular, that $F_{i}$ and $F_{f(i)}$ are diffeomorphic (flat manifolds are differentiably rigid).

Now, denote $W \cap p^{-1}\left(\left\{x_{1}, \ldots, x_{k}\right\}\right)$ by $L_{W}$, where $W$ is a wall, and write also $L=\bigcup L_{W}=p^{-1}\left(\left\{x_{1}, \ldots, x_{k}\right\}\right)$. Now, because $f\left(x_{i}\right)=x_{f(i)}$ and $f_{*}\left(\pi_{1}\left(F_{i}, x_{i}\right)\right)=$ $\pi_{1}\left(F_{f(i)}, x_{f(i)}\right)$, we have that for every wall $W$ there is a wall $W^{\prime}$ such that $\tilde{f}\left(L_{W}\right)=$ $L_{W^{\prime}}$. This gives a bijection $f^{\mathcal{W}}$ from $\mathcal{W}$ to itself.

9.4. Claim. If $W_{1}$ and $W_{2}$ are walls of a region $R$ (i.e., $W_{1}, W_{2} \subset \partial R$ ), then $W_{1}^{\prime}=f^{\mathcal{W}}\left(W_{1}\right)$ and $W_{2}^{\prime}=f^{\mathcal{W}}\left(W_{2}\right)$ are also walls of a region $R^{\prime}$.

The proof of 9.4 is given in two steps.

Step 1. Let $W$ be a wall and $W^{\prime}=f^{\mathcal{W}}(W)$. We prove that there is a bounded homotopy $H: W \times[0,1] \rightarrow \mathbb{R}^{n+1}$, such that $H_{0}=\left.\tilde{f}\right|_{W}$ and $H_{1}: W \times\{1\} \rightarrow W^{\prime}$ is a diffeomorphism (a bounded homotopy means that there is a constant $s$ such that $\forall x \in W, \operatorname{diam}\{H(x,[0,1])\} \leq s)$. To see this, note that because $D M$ and $F_{i}$ are aspherical and $f_{*}\left(\pi_{1}\left(F_{i}, x_{i}\right)\right)=\pi_{1}\left(F_{f(i)}, x_{f(i)}\right)$, we have that the map $\left.f\right|_{F_{i}}: F_{i} \rightarrow$ $D M$ is homotopic, by a homotopy $H^{\prime}$, to a diffeomorphism $h^{\prime}: F_{i} \rightarrow F_{f(i)} \subset D M$ 
with $h_{*}^{\prime}=\left.f_{*}\right|_{\pi_{1}\left(F_{i}\right)}$. Then lifting $H^{\prime}$ and $h^{\prime}$, we obtain $H$ and $h$, and because $D M$ is compact, $H^{\prime}$ and $H$ are bounded.

Step 2. We complete the proof. Take $W_{1}$ and $W_{2}$ walls of a region $R$. This is equivalent to saying that there is no wall separating them. Write $W_{i}^{\prime}=f^{\mathcal{W}}\left(W_{i}\right), i=$ 1,2 . If $W_{1}^{\prime}$ and $W_{2}^{\prime}$ are not walls of a region, then there is a wall $W_{0}^{\prime}=f^{\mathcal{W}}\left(W_{0}\right)$ separating them. By step $1,\left.\tilde{f}\right|_{W_{0}}$ can be deformed to a diffeomorphism $h: W_{0} \rightarrow W_{0}^{\prime}$ by a homotopy of finite diameter $s$. Let $z_{i} \in L_{W_{i}}$ be such that for $y_{i}=\tilde{f}\left(z_{i}\right) \in$ $L_{W_{i}^{\prime}}, i=1,2$, we have $d\left(y_{i}, W_{0}^{\prime}\right)>s$ (we are using the claim in lemma 9.2 here). Then, because $W_{0}$ does not separate $z_{1}$ and $z_{2}, \tilde{f}\left(W_{0}\right)$ does not separate $\tilde{f}\left(z_{1}\right)=y_{1}$ and $\tilde{f}\left(z_{2}\right)=y_{2}$. But then, because $W_{0}^{\prime}$ and $\tilde{f}\left(W_{0}\right)$ are $s$-closed and $d\left(y_{i}, W_{0}^{\prime}\right)>s$, we get that $W_{0}^{\prime}$ also does not separate $y_{1}$ and $y_{2}$, which is a contradiction.

Then we can join $y_{1}$ and $y_{2}$ by a path $\gamma$ that does not intersect $\tilde{f}\left(W_{0}\right)\left(W_{0}\right.$ does not separate $W_{1}$ and $\left.W_{2}\right)$ and $f \gamma$ is homotopic rel\{0,1\} to a path $\gamma^{\prime}$ that intersects $W_{0}^{\prime}$ once $\left(W_{0}^{\prime}\right.$ does separate $W_{1}^{\prime}$ and $\left.W_{2}^{\prime}\right)$. This is a contradiction since the bounded homotopy between $\tilde{f}\left(W_{0}\right)$ and $W_{0}^{\prime}$ does not pass through $y_{1}, y_{2}$, and $\tilde{f} \gamma \cap \tilde{f}\left(W_{0}\right)=\phi$. This completes the proof of claim 9.4. We now complete the proof of theorem 2.

First we see that claim 9.4 implies

9.5. $f_{*}\left(\left(j_{i}\right)_{*}\left(\pi_{1}\left(M^{0}\right)\right)\right)=\left(j_{i^{\prime}}\right)_{*}\left(\pi_{1}\left(M^{0}\right)\right)$, where we have either $i=i^{\prime}$ or $i \neq i^{\prime}$ for $i, i^{\prime}=1,2$.

To see this take, for instance, a closed path $\gamma \in \pi_{1}\left(j_{1}\left(M^{0}\right)\right)$. Then lifting $\gamma$ we obtain a path $\tilde{\gamma}:[0,1] \rightarrow \mathbb{R}^{n}$ that joins two walls and such that $\tilde{\gamma}(0,1)$ does not intersect any walls. Claim 9.4 implies $\tilde{f}(\tilde{\gamma}(0))$ and $\tilde{f}(\tilde{\gamma}(1))$ lie on walls of a region $R^{\prime}$, so that they can be joined by a path $\alpha:[0,1] \rightarrow \mathbb{R}^{n}$ with $\alpha(0,1) \subset R^{\prime}$. But $\alpha \sim \tilde{f} \tilde{\gamma}$ rel $\{0,1\}$, which means that $f_{*}[\tilde{\gamma}]$ belongs to $\left(j_{i}\right)_{*} \pi_{1}\left(M^{0}\right)$ for $i$ such that $p\left(R^{\prime}\right)=j_{i}\left(M^{0}\right)$. This proves 9.5 .

Write $\sigma_{i}=\left.f_{*}\right|_{\pi_{1}\left(j_{i}\left(M^{0}\right)\right)}: \pi_{1}\left(j_{i}\left(M^{0}\right)\right) \rightarrow \pi_{1}\left(j_{i^{\prime}}\left(M^{0}\right)\right)$. By Mostow's rigidity theorem, there are isometries $g_{i}: M \rightarrow M, i=1,2$, inducing (up to conjugation) the $\sigma_{i}$ 's. But isometries send cusps to cusps and because any isometry of $F_{i} \times[0, \infty)$, with metric $e^{-2 t} S+d t^{2}$, is clear to preserve, eventually, the $t$-levels (the radius of injectivity is a strictly decreasing function of $t$ ), we see that we can glue $g_{1}$ and $g_{2}$ obtaining a diffeomorphism $g: D M \rightarrow D M$, such that $f_{*}=g_{*}$ (note that conjugation is not a problem: just move the base point around the conjugating path using an isotopy that leaves the far end of the cusps invariant). But $D M$ is aspherical, and hence $f \sim g$. This completes the proof of theorem 2 .

Proof of theorem 2A. By [8] $f$ is homotopic to a homeomorphism (for dimensions greater than four). The proof of theorem 2 above now follows word by word.

9.6. Corollary. Let $\Sigma$ be a PL structure on $D M$, $\operatorname{dim} M>4$, non-concordant to the canonical one. Then $(D M, \Sigma)$ is not $P L$ equivalent to $D M$.

Proof. Let $f:(D M, \Sigma) \rightarrow D M$ be a $P L$ homeomorphism. We have two cases:

Particular Case. Suppose $f$ is homotopic to the identity. Denote by $H$ such homotopy and write $H: f \sim I d_{D M}$. Then $\bar{H}: f \times I d_{[0,1]} \sim I d_{D M \times[0,1]}$, where $\bar{H}=H \times I d_{[0,1]}$. 
Since $\bar{H}$ restricts to a homeomorphism on the boundary of $D M \times[0,1]$, we may apply (1.6.1) of [8] to get a homotopy (which is constant on $\partial(D M \times[0,1]))$ of $\bar{H}$ to a homeomorphism $\tilde{H}: D M \times[0,1] \rightarrow D M \times[0,1]$.

Because $\tilde{H}_{1}=I d$ and $\tilde{H}_{0}=f$, by pulling back the triangulation of $D M \times[0,1]$ using $\tilde{H}$, we obtain a concordance between $\tau$ and $\tau_{0}$.

General Case. The general case follows by applying theorem 2 to $f g^{-1} \sim I d_{D M}$.

\section{APPENDIX}

We give a proof of lemma 2.4:

2.4. Lemma. Let $A$ be a Riemannian metric on $M \times I$ satisfying (2.1) and $f$ as in (2.3). Then given $\epsilon>0$, there is an $L$ such that, for $\alpha>L$,

$$
-k_{0}-\epsilon<K\left(A_{\alpha}\right)<-k_{1}+\epsilon .
$$

Proof. First we prove the following.

a.1. Claim. If $A$ is any metric on $M \times I$ satisfying (2.1), then for any $\left(x_{0}, t_{0}\right) \in$ $M \times I$, there is a coordinate system $\left(x_{1}^{\alpha}, \ldots, x_{n}^{\alpha}\right)$ for $M$ near $x_{0}$ and a linear coordinate $t^{\alpha}$ for $[a, b]$ near $t_{0}$, such that

(a) $A_{\alpha}=\sum g_{i j}^{\alpha} d x_{i}^{\alpha} d x_{j}^{\alpha}+d\left(t^{\alpha}\right)^{2}$ where $g_{i j}^{\alpha}=A_{\alpha}\left(\frac{\partial}{\partial x_{i}^{\alpha}}, \frac{\partial}{\partial x_{j}^{\alpha}}\right)$.

(b) $g^{\alpha}\left(x_{0}, t_{0}\right)=I$ where $I$ is the identity matrix.

(c) $\lim _{\alpha \rightarrow \infty} \frac{\partial g^{\alpha}}{\partial t^{\alpha}}\left(x_{0}, t_{0}\right)=2 \ell_{1} I$,

$$
\begin{aligned}
& \lim _{\alpha \rightarrow \infty} \frac{\partial^{2} g^{\alpha}}{\partial\left(t^{\alpha}\right)^{2}}\left(x_{0}, t_{0}\right)=2\left(\ell_{1}^{2}+\ell_{2}\right) I \\
& \lim _{\alpha \rightarrow \infty} \frac{\partial g^{\alpha}}{\partial x_{i}^{\alpha}}\left(x_{0}, t_{0}\right)=\lim _{\alpha \rightarrow \infty} \frac{\partial^{2} g^{\alpha}}{\partial x_{i}^{\alpha} \partial x_{j}^{\alpha}}\left(x_{0}, t_{0}\right)=\lim _{\alpha \rightarrow \infty} \frac{\partial^{2} g^{\alpha}}{\partial x_{i}^{\alpha} \partial\left(t^{\alpha}\right)^{2}}\left(x_{0}, t_{0}\right) \\
& =0
\end{aligned}
$$

and all limits are uniform on $\left(x_{0}, t_{0}\right)$.

That is, all metrics satisfying (2.1) have coordinate systems such that the metrics $A_{\alpha}$ in these coordinate systems (at $\left(x_{0}, t_{0}\right)$ ), in the limit, depend only on $\ell_{1}$ and $\ell_{2}$ (up to second derivatives). Note that these limits depend just on $f$.

To prove the claim, take normal coordinates $\left(x_{1}, \ldots, x_{n}\right)$ for $M$ with respect to $\left.A\right|_{M \times\left\{t_{0}\right\}}$.

Then we have that the coordinate system $\left(x_{1}, \ldots, x_{n}, t\right)$ near $\left(x_{0}, t_{0}\right)$ satisfies:

\section{a.2.}

(a) $A=\sum g_{i j} d x_{i} d x_{j}+d t^{2}$.

(b) $g\left(x_{0}, t_{0}\right)=I$.

(c) There is a $C>0$, independent of $\left(x_{0}, t_{0}\right)$, such that

$$
\left|\frac{\partial^{k+s} g_{i j}}{\partial x_{i_{1}} \ldots \partial x_{i_{k}} \partial t^{s}}\right|<C \quad k+s \leq 2 \quad k, s \geq 0 .
$$

Note that the fact that $C$ is independent of $\left(x_{0}, t_{0}\right)$ follows from the compactness of $M \times I$.

Now define new coordinates $\left(x_{1}^{\alpha}, \ldots, x_{n}^{\alpha}, t^{\alpha}\right)$ as follows:

$$
t^{\alpha}=\alpha t, \quad x_{i}^{\alpha}=f_{\alpha}\left(t_{0}^{\alpha}\right) x_{i}, \quad \text { where } t_{0}^{\alpha}=\alpha t_{0} .
$$

Then

$$
d t^{\alpha}=\alpha d t, \quad d x_{i}^{\alpha}=f_{\alpha}\left(t_{0}^{\alpha}\right) d x_{i}
$$


and

$$
\frac{\partial}{\partial t^{\alpha}}=\frac{1}{\alpha} \frac{\partial}{\partial t}, \quad \frac{\partial}{\partial x_{i}^{\alpha}}=\frac{1}{f_{\alpha}\left(t_{0}^{\alpha}\right)} \frac{\partial}{\partial x_{i}} .
$$

We now verify that this is the coordinate system we want. (a) and (b) of the claim are direct. For (c) we make all the calculations explicitly.

We have that

$$
g^{\alpha}=\left(\frac{f_{\alpha}\left(t^{\alpha}\right)}{f_{\alpha}\left(t_{0}^{\alpha}\right)}\right)^{2} g
$$

First Limit.

$$
\begin{aligned}
\frac{\partial}{\partial t^{\alpha}}\left(g^{\alpha}\right) & =\frac{1}{\alpha} \frac{\partial}{\partial t}\left(g^{\alpha}\right) \\
& =\frac{1}{\alpha} \frac{\partial}{\partial t}\left[\left(\frac{f_{\alpha}\left(t^{\alpha}\right)}{f_{\alpha}\left(t_{0}^{\alpha}\right)}\right)^{2} g\right]=\frac{1}{\alpha}\left[\frac{2 \alpha f_{\alpha}(\alpha t) f_{\alpha}^{\prime}(\alpha t)}{f_{\alpha}^{2}\left(\alpha t_{0}\right)} g+\left(\frac{f_{\alpha}\left(t^{\alpha}\right)}{f_{\alpha}\left(t_{0}^{\alpha}\right)}\right)^{2} \frac{\partial g}{\partial t}\right] .
\end{aligned}
$$

At $(x, t)=\left(x_{0}, t_{0}\right)$, we have (by (b) of 2.3 and (c) of a.2)

$$
\frac{\partial g^{\alpha}}{\partial t^{\alpha}}\left(x_{0}, t_{0}\right)=\frac{1}{\alpha}\left[\frac{2 \alpha f_{\alpha}^{\prime}\left(\alpha t_{0}\right)}{f_{\alpha}\left(\alpha t_{0}\right)} I+\frac{\partial g}{\partial t}\right] \rightarrow 2 \ell_{1} I .
$$

Second Limit.

$$
\begin{aligned}
\frac{\partial^{2} g^{\alpha}}{\partial\left(t^{\alpha}\right)^{2}}=\frac{\partial}{\partial t^{\alpha}}\left\{\frac{1}{\alpha}\left[\frac{2 \alpha f_{\alpha}(\alpha t) f_{\alpha}^{\prime}(\alpha t)}{f_{\alpha}^{2}\left(\alpha t_{0}\right)} g+\left(\frac{f_{\alpha}\left(t^{\alpha}\right)}{f_{\alpha}\left(t_{0}^{\alpha}\right)}\right)^{2} \frac{\partial g}{\partial t}\right]\right\} \\
=\frac{1}{\alpha^{2}}\left\{2 \alpha^{2}\left[\frac{\left(f_{\alpha}^{\prime}(\alpha t)\right)^{2}+f_{\alpha}(\alpha t) f_{\alpha}^{\prime \prime}(\alpha t)}{f_{\alpha}^{2}\left(\alpha t_{0}\right)}\right] g\right. \\
\left.+2 \alpha\left[\frac{f_{\alpha}(\alpha t) f_{\alpha}^{\prime}(\alpha t)}{f_{\alpha}^{2}\left(\alpha t_{0}\right)}\right] \frac{\partial g}{\partial t}+\left[\frac{f_{\alpha}\left(t^{\alpha}\right)}{f_{\alpha}\left(t_{0}^{\alpha}\right)}\right]^{2} \frac{\partial^{2} g}{\partial t^{2}}\right\} .
\end{aligned}
$$

At $(x, t)=\left(x_{0}, t_{0}\right)$, we have

$$
\begin{aligned}
\frac{\partial^{2} g^{\alpha}}{\partial\left(t^{\alpha}\right)^{2}}\left(x_{0}, t_{0}\right)= & 2\left\{\left[\frac{f_{\alpha}^{\prime}\left(\alpha t_{0}\right)}{f_{\alpha}\left(\alpha t_{0}\right)}\right]^{2}+\left[\frac{f_{\alpha}^{\prime \prime}\left(\alpha t_{0}\right)}{f_{\alpha}\left(\alpha t_{0}\right)}\right]\right\} I \\
& +\frac{2}{\alpha}\left[\frac{f_{\alpha}^{\prime}\left(\alpha t_{0}\right)}{f_{\alpha}\left(\alpha t_{0}\right)}\right] \frac{\partial g}{\partial t}+\frac{1}{\alpha^{2}} \frac{\partial^{2} g}{\partial t^{2}} \rightarrow 2\left(\ell_{1}^{2}+\ell_{2}\right) I .
\end{aligned}
$$

Third Limit.

$$
\frac{\partial g^{\alpha}}{\partial x_{i}^{\alpha}}=\frac{1}{f_{\alpha}\left(\alpha t_{0}\right)} \frac{\partial}{\partial x_{i}}\left[\left(\frac{f_{\alpha}\left(t^{\alpha}\right)}{f_{\alpha}\left(t_{0}^{\alpha}\right)}\right)^{2} g\right] .
$$

At $(x, t)=\left(x_{0}, t_{0}\right)$, we have

\section{Fourth Limit.}

$$
\frac{\partial g^{\alpha}}{\partial x_{i}^{\alpha}}\left(x_{0}, t_{0}\right)=\frac{1}{f_{\alpha}\left(\alpha t_{0}\right)} \frac{\partial g}{\partial x_{i}} \rightarrow 0 .
$$

$$
\frac{\partial^{2} g^{\alpha}}{\partial x_{i}^{\alpha} \partial\left(t^{\alpha}\right)}\left(x_{0}, t_{0}\right)=\frac{1}{f_{\alpha}\left(\alpha t_{0}\right)}\left[\frac{2 f_{\alpha}^{\prime}\left(\alpha t_{0}\right)}{f_{\alpha}\left(\alpha t_{0}\right)} \frac{\partial g}{\partial x_{i}}+\frac{\partial^{2} g}{\partial t \partial x_{i}}\right] \rightarrow 0
$$

Fifth Limit.

$$
\frac{\partial^{2} g^{\alpha}}{\partial x_{i}^{\alpha} \partial x_{j}^{\alpha}}\left(x_{0}, t_{0}\right)=\frac{1}{f_{\alpha}^{2}\left(\alpha t_{0}\right)} \frac{\partial^{2} g}{\partial x_{i} \partial x_{j}} \rightarrow 0 .
$$

That completes the proof of the claim. 
We now complete the proof of the lemma. Let $S$ be any metric on $M$ and write $B=S+d t^{2}$. Let $P$ be a 2 -plane with orthonormal basis $\left\{v_{1}, v_{2}\right\}$ and write

$$
v_{i}=\sum a_{i j} \frac{\partial}{\partial y_{j}^{\alpha}}+a_{i} \frac{\partial}{\partial t^{\alpha}},
$$

where $\left(y_{1}^{\alpha}, \ldots, y_{n}^{\alpha}, t^{\alpha}\right)$ are coordinates for $B$ as in the claim.

Set

$$
v_{i}^{\prime}=\sum a_{i j} \frac{\partial}{\partial x_{j}^{\alpha}}+a_{i} \frac{\partial}{\partial t^{\alpha}}
$$

and denote by $P^{\prime \prime}$ the 2-plane spanned by $\left\{v_{1}^{\prime}, v_{2}^{\prime}\right\}$. Now, $K_{P}\left(B_{\alpha}\right)$ and $K_{P^{\prime}}\left(A_{\alpha}\right)$ are polynomials in $g_{i j}^{\alpha}$ and its partial derivatives (up to second order), with the same coefficients (depending on the $a_{i j}$ ). It follows that

$$
\lim _{\alpha \rightarrow \infty}\left[K_{P}\left(B_{\alpha}\right)-K_{P^{\prime}}\left(A_{\alpha}\right)\right]=0 \quad \text { uniformly on } P \text {. }
$$

Because $B$ is a product metric, $B_{\alpha}$ is a warped metric and [3] gives the curvature

$$
K_{P}\left(B_{\alpha}\right)=-\frac{f_{\alpha}^{\prime \prime}}{f_{\alpha}} \lambda^{2}+\frac{S(v, w)-\left(f_{\alpha}^{\prime}\right)^{2}}{f_{\alpha}^{2}}\|v\|^{2}
$$

where $\left\{\lambda\left(\frac{\partial}{\partial t}\right)_{t}+v_{p}, w_{p}\right\}$, with $v_{p}, w_{p} \in T_{p} M$ is an orthonormal basis for $P$ and $B(v, w)$ is the curvature of $B$. In any case, because $f_{\alpha} \rightarrow \infty$ as $\alpha \rightarrow \infty$, we have that $k_{0}-\frac{\epsilon}{2}<K_{P}\left(B_{\alpha}\right)<k_{1}+\frac{\epsilon}{2}$, for $\alpha$ large enough.

So, if $L$ is such that $\left|K_{P}\left(B_{\alpha}\right)-K_{P^{\prime}}\left(A_{\alpha}\right)\right|<\frac{\epsilon}{2}$, for all $\alpha>L$, for all $\left(x_{0}, t_{0}\right)$ and for all 2-planes $P$, the lemma follows.

\section{REFERENCES}

1. C.S. Aravinda and F.T. Farrell, Rank 1 aspherical manifolds which do not support any nonpositively curved metric, Comm. in Analysis and Geometry 2 (1994) 65-78. MR 96e:53045

2. R. Benedetti and C. Petronio, Lectures on Hyperbolic Geometry, Universitex, Springer-Verlag, New York (1991). MR 94e:57015

3. R.L. Bishop and B. O'Neil, Manifolds of negative curvature, Trans. Amer. Math. Soc. 145 (1969) 1-49. MR 40:4891

4. A. Borel, Compact Clifford-Klein forms of symmetric spaces, Topology 2 (1963) 111-122. MR 26:3823

5. A. Borel, Introduction Aux Groupes Arithmetiques, Hermann, Paris (1969). MR 39:5577

6. M. Bridson and A. Haeflinger, Metric spaces of non-positive curvature, Springer-Verlag (1999). MR 2000k:53038

7. F.T. Farrell and L.E. Jones, Negatively curved manifolds with exotic smooth structures, J. Amer. Math. Soc. 2 (1989) 899-908. MR 90f:53075

8. F.T. Farrell and L.E. Jones, Rigidity in geometry and topology, Proceedings of the International Congress of Mathematicians, Kyoto, Japan (1990). MR 93g:57041

9. F.T. Farrell and L.E. Jones, Exotic smoothings of hyperbolic manifolds which do not support pinched negative curvature, Proc. Amer. Math. Soc. 121 (1994) 627-630. MR 94h:57047

10. F. T. Farrell, L. E. Jones and P. Ontaneda, Hyperbolic manifolds with negatively curved exotic triangulations in dimensions larger than five, Jour. Diff. Geom. 48 (1998) 319-322. MR 2000f:57003

11. D. Gromoll and J.A. Wolf, Some relations between the metric structure and the algebraic structure of the fundamental group in manifolds of non-positive curvature, Bull. Amer. Math. Soc. 77 (1971) 545-552. MR 43:6841

12. R.C. Kirby and L.C. Siebenmann, Foundational Essays on Topological Manifolds, Smoothings, and Triangulations, Annals of Math. Studies, 88, Princeton University Press, Princeton (1977). MR 58:31082

13. H.B. Lawson and S.T. Yau, Compact manifolds of nonpositive curvature, J. Diff. Geom. 7 (1972) 211-228. MR 48:12402 
14. J.J. Millson, On the first Betti number of a constant negatively curved manifold, Ann. of Math. 104 (1976) 235-247. MR 54:10488

15. P. Ontaneda, Hyperbolic manifolds with negatively curved exotic triangulations in dimension six, Jour. Diff. Geom. 40 (1994) 7-22. MR 95d:57013

16. C. P. Rourke and B. J. Sanderson, Introduction to piecewise-linear topology, Springer-Verlag, (1972). MR 50:3236

17. C.T.C. Wall, Surgery on Compact Manifolds, Academic Press, London (1971). MR 55:4217

Departamento de Matematica, Universidade Federal de Pernambuco, Cidade UniverSitARia, Recife, PE 50670-901, BraziL

E-mail address: ontaneda@dmat.ufpe.br 